\title{
Homotopy groups of the moduli space of metrics of positive scalar curvature
}

\author{
BORIS BOTVINNIK \\ BERNHARD HANKE \\ THOMAS SCHICK \\ MARK WALSH
}

\begin{abstract}
We show by explicit examples that in many degrees in a stable range the homotopy groups of the moduli spaces of Riemannian metrics of positive scalar curvature on closed smooth manifolds can be non-trivial. This is achieved by further developing and then applying a family version of the surgery construction of Gromov-Lawson to certain nonlinear smooth sphere bundles constructed by Hatcher.
\end{abstract}

$53-02 ; 55-02$

\section{Introduction}

\subsection{Motivation}

Let $M$ be a closed smooth manifold. In this article we study the topology of the space of metrics of positive scalar curvature $\operatorname{Riem}^{+}(M)$ and of corresponding moduli spaces. We abbreviate "metric of positive scalar curvature" by "psc-metric".

It has been known for a long time that there are quite a few obstructions to the existence of psc-metrics. This starts in dimension 2, where the Gauß-Bonnet theorem tells us that only the sphere and $\mathbb{R} P^{2}$ admit such a metric. In general the Lichnerowicz formula in combination with the Atiyah-Singer index theorem implies that if $M$ is a spin manifold and admits a psc-metric, then the $\hat{A}$-genus of $M$ is zero. The GromovLawson-Rosenberg conjecture (see Rosenberg [25]) was an attempt to completely characterize those spin manifolds admitting psc-metrics. It was later disproved by Schick [26].

In spite of the complicated picture for general manifolds, the existence question has been resolved completely for simply connected manifolds $M$ of dimension at least five. Gromov and Lawson proved in [12] that if $M$ is not spin, then there is no obstruction and $M$ admits a psc-metric. Assuming that $M$ is spin, Stolz [27] proved that the only obstruction is the $K O$-valued index of the Dirac operator on $M$. 
If $M$ admits a psc-metric, one can go on and investigate the topology of $\operatorname{Riem}^{+}(M)$, the space of psc-metrics on $M$ equipped with the the $C^{\infty}$-topology. Note that $\operatorname{Diff}(M)$, the diffeomorphism group of $M$, acts on $\mathcal{R i e m}^{+}(M)$ via pull-back, and so it is even more natural to study the moduli space $\mathcal{R i e m}^{+}(M) / \operatorname{Diff}(M)$.

In the spin case index theoretic methods were used to show that the spaces $\mathcal{R i e m}^{+}(M)$ and $\mathcal{R i e m}^{+}(M) / \operatorname{Diff}(M)$ have infinitely many components in many cases. See, for example, the work of Gromov-Lawson [13] or Lawson-Michelsohn [20] or, for more refined versions, the papers of Botvinnik and Gilkey [5], Leichtnam and Piazza [21], and Piazza and Schick [24]. If $M$ is simply connected, this applies to the case when $\operatorname{dim}(M) \equiv 1(\bmod 4)$.

Hitchin observed in his thesis [15, Theorem 4.7] that sometimes, in the spin case, nonzero elements in the homotopy groups of $\operatorname{Diff}(M)$ yield, via the action of $\operatorname{Diff}(M)$ on $\mathcal{R i e m}^{+}(M)$, non-zero elements in the homotopy groups of $\mathcal{R i e m}^{+}(M)$. More precisely, he proves this way that $\pi_{0}\left(\operatorname{Riem}^{+}\left(M^{n}\right)\right)$ is non-trivial for $n \equiv-1,0,1$ $(\bmod 8)$ and $\pi_{1}\left(\mathcal{R i e m}^{+}\left(M^{n}\right)\right)$ is non-trivial for $n \equiv-1,0(\bmod 8)$.

Contrasting these positive results, it has been an open problem to decide whether the groups $\pi_{k}\left(\operatorname{Riem}^{+}(M)\right)$ for $k>1$ or $\pi_{k}\left(\mathcal{R i e m}^{+}(M) / \operatorname{Diff}(M)\right)$ for $k>0$ can be non-trivial. Note that, by construction, Hitchin's elements in $\pi_{k}\left(\mathcal{R i e m}^{+}\left(S^{n}\right)\right)$, $k=0,1$, are mapped to zero in the moduli space $\mathcal{R i e m}^{+}(M) / \operatorname{Diff}(M)$. Some experts even raised the suspicion that the components of this moduli space are always contractible.

\subsection{Moduli spaces of psc-merics}

In this paper we will construct many examples of non-zero elements in higher homotopy groups of moduli spaces of psc-metrics on closed smooth manifolds $M$. We denote by $\operatorname{Riem}(M)$ the space of all Riemannian metrics with the $C^{\infty}$-topology. The group of diffeomorphisms $\operatorname{Diff}(M)$ acts from the right on the space $\operatorname{Riem}(M)$ by pull-back: $(g, \phi) \mapsto \phi^{*}(g)$. The orbit space of this action is the moduli space of Riemannian metrics on $M$ and written $\mathcal{M}(M)$. The orbit space $\mathcal{M}^{+}(M)$ of the restricted $\operatorname{Diff}(M)$-action on the subspace $\mathcal{R i e m}^{+}(M)$ of psc-metrics, the moduli space of Riemannian metrics of positive scalar curvature on $M$, is our principal object of interest.

In general the action of the full diffeomorphism group is not free on $\mathcal{R} \operatorname{iem}(M)$ : For example, if a finite group $G$ acts effectively on $M$ (that is, if $G$ occurs as a finite subgroup of $\operatorname{Diff}(M))$, then any metric on $M$ can be averaged over $G$, and the resulting metric will be fixed by $G$. Therefore we also consider the moduli spaces with observer as proposed by Akutagawa and Botvinnik [1]. 
Definition 1.1 Let $\left(M, x_{0}\right)$ be a connected closed smooth manifold with some basepoint $x_{0}$. Let $\operatorname{Diff}_{x_{0}}(M)$ be the subgroup of $\operatorname{Diff}(M)$ of those diffeomorphisms which fix $x_{0}$ and induce the identity on the tangent space $T_{x_{0}} M$. This is the group of diffeomorphisms which preserve an observer based at $x_{0}$.

Lemma 1.2 If $\left(M, x_{0}\right)$ is a connected smooth closed manifold with a basepoint $x_{0}$ then $\operatorname{Diff}_{x_{0}}(M)$ acts freely on the space $\mathcal{R} \operatorname{iem}(M)$ of Riemannian metrics on $M$.

Proof This lemma is well known, compare, for example, Bourguignon [6, Proposition IV.5]. For convenience we recall the proof. Assume $g$ is a Riemannian metric on $M$, $\phi \in \operatorname{Diff}_{x_{0}}(M)$ and $\phi^{*} g=g$. This means that the map $\phi$ is an isometry of $(M, g)$. As $x_{0}$ and $T_{x_{0}} M$ are fixed by $\phi$, so are all geodesics emenating from $x_{0}$ (pointwise). Since $M$ is closed and connected, every point lies on such a geodesic, so $\phi$ is the identity.

In the following we equip $\operatorname{Diff}(M)$ and $\operatorname{Diff}_{x_{0}}(M)$ with the $C^{\infty}$-topologies. Let $\mathcal{M}_{x_{0}}(M)=\mathcal{R} \operatorname{iem}(M) / \operatorname{Diff}_{x_{0}}(M)$. We call $\mathcal{M}_{x_{0}}(M)$ the observer moduli space of Riemannian metrics on $M$. Since the space $\operatorname{Riem}(M)$ is contractible and the action of $\operatorname{Diff}_{x_{0}}(M)$ on $\mathcal{R i e m}(M)$ is proper (see Ebin [8]), Lemma 1.2 implies that the orbit space $\mathcal{M}_{x_{0}}(M)$ is homotopy equivalent to the classifying space $B \operatorname{Diff}_{x_{0}}(M)$ of the group Diff $x_{x_{0}}(M)$. In particular one obtains a Diff $x_{0}(M)$-principal fiber bundle

$$
\operatorname{Diff}_{x_{0}}(M) \rightarrow \mathcal{R i e m}(M) \rightarrow \mathcal{M}_{x_{0}}(M)
$$

This yields isomorphisms of homotopy groups

$$
\pi_{q} \mathcal{M}_{x_{0}}(M)=\pi_{q} B \operatorname{Diff}_{x_{0}}(M) \cong \pi_{q-1} \operatorname{Diff}_{x_{0}}(M), \quad q \geq 1 .
$$

Now we restrict the action of $\operatorname{Diff}_{x_{0}}(M)$ to the subspace $\mathcal{R i e m}^{+}(M)$ of psc-metrics. Clearly this action is free as well. We call the orbit space

$$
\mathcal{M}_{x_{0}}^{+}(M):=\mathcal{R i e m}^{+}(M) / \operatorname{Diff}_{x_{0}}(M)
$$

the observer moduli space of psc-metrics. Again we obtain a Diff $x_{x_{0}}(M)$-principal fiber bundle

$$
\operatorname{Diff}_{x_{0}}(M) \rightarrow \mathcal{R i e m}^{+}(M) \rightarrow \mathcal{M}_{x_{0}}^{+}(M) .
$$

The inclusion $\operatorname{Riem}^{+}(M) \hookrightarrow \mathcal{R} \operatorname{iem}(M)$ induces inclusions of moduli spaces

$$
\mathcal{M}^{+}(M) \hookrightarrow \mathcal{M}(M) \text { and } \mathcal{M}_{x_{0}}^{+}(M) \hookrightarrow \mathcal{M}_{x_{0}}(M) .
$$

We collect our observations in the following lemma. 
Lemma 1.3 Let $M$ be a connected closed manifold and $x_{0} \in M$. Then

(a) there is the following commutative diagram of principal $\operatorname{Diff}_{x_{0}}(M)$-fibrations

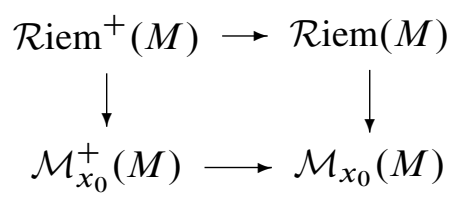

(b) the observer moduli space $\mathcal{M}_{x_{0}}(M)$ of Riemannian metrics on $M$ is homotopy equivalent to the classifying space $B \operatorname{Diff}_{x_{0}}(M)$;

(c) there is a homotopy fibration

$$
\operatorname{Riem}^{+}(M) \rightarrow \mathcal{M}_{x_{0}}^{+}(M) \rightarrow \mathcal{M}_{x_{0}}(M) .
$$

The constructions of Hitchin [15] use certain non-zero elements in $\pi_{k} \operatorname{Diff}(M)$ and push them forward to the space $\mathcal{R i e m}^{+}(M)$ via the first map in (2). It is then shown that these elements are non-zero in $\pi_{k} \mathcal{R i e m}^{+}(M)$ (for $k=0,1$ ).

Our main method will be similar, but starting from the fiber sequence (4). We will show that certain non-zero elements of $\pi_{k} B \operatorname{Diff}_{x_{0}}(M)=\pi_{k} \mathcal{M}_{x_{0}}(M)$ can be lifted to $\mathcal{M}_{x_{0}}^{+}(M)$. Once such lifts have been constructed, it is immediate that they represent non-zero elements in $\pi_{k} \mathcal{M}_{x_{0}}^{+}(M)$ as their images are non-zero in $\pi_{k} \mathcal{M}_{x_{0}}(M)$.

\subsection{The results}

We start from the particular manifold $M=S^{n}$. Let $x_{0} \in S^{n}$ be a base point. Then the group $\operatorname{Diff}_{x_{0}}\left(S^{n}\right)$ is homotopy equivalent to the group $\operatorname{Diff}\left(D^{n}, \partial D^{n}\right)$ of diffeomorphisms of the disk $D^{n}$ which restrict to the identity on the boundary $\partial D^{n}$. These groups and their classifying spaces have been studied extensively. In particular the rational homotopy groups $\pi_{q} B \operatorname{Diff}_{x_{0}}\left(S^{n}\right) \otimes \mathbb{Q}$ are known from algebraic $K$-theory computations and Waldhausen $K$-theory in a stable range.

Theorem 1.4 (Farrell and Hsiang [9]) Let $0<k \ll n$. Then

$$
\pi_{k} B \operatorname{Diff}_{x_{0}}\left(S^{n}\right) \otimes \mathbb{Q}= \begin{cases}\mathbb{Q} & \text { if } n \text { odd }, k=4 q \\ 0 & \text { else. }\end{cases}
$$

Here and in later places the shorthand notation $k \ll n$ means that for fixed $k$ there is an $N \in \mathbb{N}$ so that the statement is true for all $n \geq N$. 
Consider the inclusion map $\iota: \mathcal{M}_{x_{0}}^{+}\left(S^{n}\right) \rightarrow \mathcal{M}_{x_{0}}\left(S^{n}\right)=B$ Diff $x_{0}\left(S^{n}\right)$ and the corresponding homomorphism of homotopy groups:

$$
\iota_{*}: \pi_{k} \mathcal{M}_{x_{0}}^{+}\left(S^{n}\right) \rightarrow \pi_{k} \mathcal{M}_{x_{0}}\left(S^{n}\right) .
$$

Here is our first main result.

Theorem 1.5 The homomorphism

$$
\iota_{*} \otimes \mathbb{Q}: \pi_{k} \mathcal{M}_{x_{0}}^{+}\left(S^{n}\right) \otimes \mathbb{Q} \rightarrow \pi_{k} \mathcal{M}_{x_{0}}\left(S^{n}\right) \otimes \mathbb{Q}
$$

is an epimorphism for $0<k \ll n$. In particular, the groups $\pi_{k} \mathcal{M}_{x_{0}}^{+}\left(S^{n}\right)$ are non-trivial for odd $n$ and $0<k=4 q \ll n$.

Theorem 1.4 is essentially an existence theorem and does not directly lead to a geometric interpretation of the generators of $\pi_{k} B \operatorname{Diff}_{x_{0}}\left(S^{n}\right) \otimes \mathbb{Q}$. This was achieved later in the work of Bökstedt [4] and Igusa [16; 18] based on a construction of certain smooth nonlinear disk and sphere bundles over $S^{k}$ due to Hatcher. The nontriviality of some of these bundles is detected by the non-vanishing of a higher Franz-Reidemeister torsion invariant.

Recall from Igusa $[16 ; 17 ; 18]$ that for any closed smooth manifold $M$ there are universal higher Franz-Reidemeister torsion classes $\tau_{2 q} \in H^{4 q}(B \operatorname{Torr}(M)$; $\mathbb{Q})$, where $\operatorname{Torr}(M) \subset \operatorname{Diff}(M)$ is the subgroup of diffeomorphisms of $M$ that act trivially on $H_{*}(M ; \mathbb{Q})$. Note that $\operatorname{Diff}_{x_{0}}\left(S^{n}\right) \subset \operatorname{Torr}\left(S^{n}\right)$ and that $\operatorname{Torr}\left(S^{n}\right)$ is the subgroup of $\operatorname{Diff}\left(S^{n}\right)$ consisting of orientation preserving diffeomorphisms. In particular, these classes define characteristic classes for smooth fiber bundles $M \rightarrow E \rightarrow B$ over path connected closed smooth manifolds $B$ with $\pi_{1}(B)$ acting trivially on $H_{*}(M ; \mathbb{Q})$. (The last condition can be weakened to $H_{*}(M ; \mathbb{Q})$ being a unipotent $\pi_{1}(M)$-module [18], but this is not needed here).

The relevant class $\tau_{2 q} \in H^{4 q}\left(S^{4 q} ; \mathbb{Q}\right)$ of the Hatcher bundles over $S^{4 q}$ with fiber $S^{n}$ was computed by Goette [11] and Igusa [16; 18] and shown to be non-zero, if $n$ is odd. The generators of $\pi_{k} B \operatorname{Diff}_{x_{0}}\left(S^{n}\right)$ appearing in Theorem 1.4 can be represented by classifying maps $S^{k} \rightarrow B \operatorname{Diff}_{x_{0}}\left(S^{n}\right)$ of these Hatcher bundles in this way. In order to prove Theorem 1.5 we construct families of psc-metrics on these bundles.

Therefore, in Section 2, we will first study how and under which conditions such constructions can be carried out. Assuming that a given smooth bundle admits a fiberwise Morse function, we use the surgery technique developed by Walsh [28], which generalizes the Gromov-Lawson construction of psc-metrics via handle decompositions (see Gajer [10] and Gromov and Lawson [12]), to families of Morse functions, in order to construct families of psc-metrics on this bundle, see Theorem 2.9. This is the 
technical heart of the paper at hand. Compared to Walsh [28] the novel point is the generalization of the relevant steps of this construction to nontrivial fiber bundles.

Then, we will study particular generators of $\pi_{k} B$ Diff $x_{x_{0}}\left(S^{n}\right) \otimes \mathbb{Q}$ for suitable $k$ and $n$, as in Theorem 1.4. To give a better idea how we are going to proceed, recall that the observer moduli space $\mathcal{M}_{x_{0}}\left(S^{n}\right)=B$ Diff $x_{0}\left(S^{n}\right)$ serves as a classifying space of smooth fiber bundles with fiber $S^{n}$ and structure group $\operatorname{Diff}_{x_{0}}\left(S^{n}\right)$. We obtain the universal smooth fiber bundle

$$
S^{n} \rightarrow \mathcal{R i e m}\left(S^{n}\right) \times_{\operatorname{Diff}_{x_{0}}\left(S^{n}\right)} S^{n} \rightarrow \mathcal{R} \operatorname{iem}\left(S^{n}\right) / \operatorname{Diff}_{x_{0}}\left(S^{n}\right) .
$$

In particular, a map $f: S^{k} \rightarrow B \operatorname{Diff}_{x_{0}}\left(S^{n}\right)$ representing an element $\alpha \in \pi_{k} B \operatorname{Diff}_{x_{0}}\left(S^{n}\right)$ gives rise to a commutative diagram of smooth fiber bundles

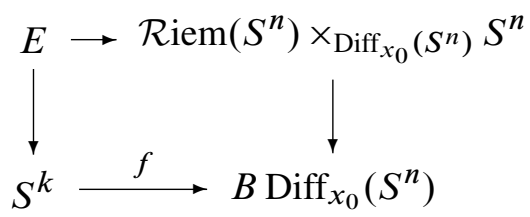

This shows that a lift of the class $\alpha \in B \operatorname{Diff}_{x_{0}}\left(S^{n}\right)$ to $\pi_{k} \mathcal{M}_{x_{0}}^{+}\left(S^{n}\right)$ is nothing but a family of psc-metrics on the fibers $E_{x}$ of the Hatcher bundle $S^{n} \rightarrow E \rightarrow S^{k}$, parameterized by $x \in S^{k}$, so as to give a smooth family of fiberwise psc-metrics on $E$.

We will explain the precise relationship in Section 3 and show that the construction described in Section 2 applies to Hatcher's $S^{n}$-bundles. Here we make use of a familiy of Morse functions on these bundles as described by Goette [11, Section 5.b]. This will finish the proof of Theorem 1.5.

Given a closed smooth manifold $M$ of dimension $n$, we can take the fiberwise connected sum of the trivial bundle $S^{k} \times M \rightarrow S^{k}$ and Hatcher's exotic $S^{n}$-bundle. Using additivity of higher torsion invariants (see Igusa [18, Section 3]) we obtain non-trivial elements in $\pi_{k} \mathcal{M}_{x_{0}}(M)$ for given $k$ for any manifold $M$ of odd dimension $n$ as long as $k \ll n$.

If in addition $M$ admits a psc-metric, this can be combined with the fiberwise pscmetric on Hatcher's $S^{n}$-bundle constructed earlier to obtain a fiberwise psc-metric on the resulting nontrivial $M$-bundle over $S^{k}$. This shows:

Theorem 1.6 Let $M$ be a closed smooth manifold admitting a metric $h$ of positive scalar curvature. If $\operatorname{dim} M$ is odd, then the homotopy groups $\pi_{k}\left(\mathcal{M}_{x_{0}}^{+}(M),[h]\right)$ are non-trivial for $0<k=4 q \ll \operatorname{dim} M$. 
In order to study the homotopy type of the classical moduli space of psc-metrics it remains to construct examples of manifolds $M$ for which the non-zero elements in $\pi_{k} \mathcal{M}_{x_{0}}^{+}(M)$ constructed in Theorem 1.6 are not mapped to zero under the canonical map $\pi_{k} \mathcal{M}_{x_{0}}^{+}(M) \rightarrow \pi_{k} \mathcal{M}^{+}(M)$. This will be done in Section 4 and leads to a proof of the following conclusive result.

Theorem 1.7 For any $d>0$ there exists a closed smooth manifold $M$ admitting a metric $h$ of positive scalar curvature so that $\pi_{4 q}\left(\mathcal{M}^{+}(M),[h]\right)$ is non-trivial for $0<q \leq d$.

Remark One should mention that the manifolds we construct in Theorem 1.7 do not admit spin structures and are of odd dimension. In particular, the usual methods to distinguish elements of $\pi_{0} \mathcal{M}^{+}(M)$, which use the index of the Dirac operator, do not apply to these manifolds, and we have no non-trivial lower bound on the number of components of $\mathcal{M}^{+}(M)$.

Remark Finding non-zero elements of $\pi_{k} \mathcal{R i e m}^{+}(M)$ for $k>1$ remains an open problem. It would be especially interesting to find examples with non-zero image in $\pi_{k}\left(\mathcal{R i e m}^{+}(M) / \operatorname{Diff}(M)\right)$, or at least in $\pi_{k}\left(\mathcal{R i e m}^{+}(M) / \operatorname{Diff}_{x_{0}}(M)\right)$.

We expect that a solution of this problem requires a different method than the one employed in Sections 3 and 4 of our paper.

\subsection{Acknowledgement}

Boris Botvinnik would like to thank K Igusa and D Burghelea for inspiring discussions on topological and analytical torsion and thank SFB-478 (Geometrische Strukturen in der Mathematik, Münster, Germany) and IHES for financial support and hospitality. Mark Walsh also would like to thank SFB-478 for financial support and hospitality. Thomas Schick was partially supported by the Courant Research Center "Higher order structures in Mathematics" within the German initiative of excellence.

\section{The surgery method in twisted families}

The aim of this section is to prove a result on the construction of fiberwise pcs-metrics on certain smooth fiber bundles. At first we briefly review the Gromov-Lawson surgery technique [12] on a single manifold. Here we use the approach developed by Walsh [28; 29]. 


\subsection{Review of the surgery technique on a single manifold}

Let $W$ be a compact manifold with non-empty boundary $\partial W$ and with $\operatorname{dim} W=n+1$. We assume that the boundary $\partial W$ is the disjoint union of two manifolds $\partial_{0} W$ and $\partial_{1} W$ both of which come with collars

$$
\partial_{0} W \times[0, \epsilon) \subset W, \quad \partial_{1} W \times(1-\epsilon, 1] \subset W,
$$

where $\epsilon$ is taken with respect to some fixed reference metric $\mathfrak{m}$ on $W$, see Definition 2.1 below. By a Morse function on $W$ we mean a Morse function $f: W \rightarrow[0,1]$ such that

$$
f^{-1}(0)=\partial_{0} W, \quad f^{-1}(1)=\partial_{1} W
$$

and the restriction of $f$ to the collars (6) coincides with the projection onto the second factor

$$
\partial_{0} W \times[0, \epsilon) \rightarrow[0, \epsilon), \quad \partial_{1} W \times(1-\epsilon, 1] \rightarrow(1-\epsilon, 1] .
$$

We denote by $\operatorname{Cr}(f)$ the set of critical points of $f$.

We say that a Morse function $f: W \rightarrow[0,1]$ is admissible if all its critical points have indices at most $(n-2)$ (where $\operatorname{dim} W=n+1)$. We note that the last condition is equivalent to the "codimension at least three" requirement for the Gromov-Lawson surgery method. We denote by $\operatorname{Morse}(W)$ and $\operatorname{Morse}^{\text {adm }}(W)$ the spaces of Morse functions and admissible Morse functions, respectively, which we equip with the $C^{\infty}$-topologies.

Definition 2.1 Let $f \in \operatorname{Morse}^{\operatorname{adm}}(W)$. A Riemannian metric $\mathfrak{m}$ on $W$ is compatible with the Morse function $f$ if for every critical point $p \in \operatorname{Cr}(f)$ with ind $p=\lambda$ the positive and negative eigenspaces $T_{p} W^{+}$and $T_{p} W^{-}$of the Hessian $d^{2} f$ are $\mathfrak{m}$-orthogonal, and $\left.d^{2} f\right|_{T_{p} W^{+}}=\left.\mathfrak{m}\right|_{T_{p} W^{+}},\left.d^{2} f\right|_{T_{p} W^{-}}=-\left.\mathfrak{m}\right|_{T_{p} W^{-}}$.

We notice that for a given Morse function $f$, the space of compatible metrics is convex. Thus the space of pairs $(f, \mathfrak{m})$, where $f \in \operatorname{Morse}^{\operatorname{adm}}(W)$, and $\mathfrak{m}$ is a metric compatible with $f$, is homotopy equivalent to the space $\operatorname{Morse}^{\operatorname{adm}}(W)$. We call a pair $(f, \mathfrak{m})$ as above an admissible Morse pair. We emphasize that the metric $\mathfrak{m}$ on $W$ has no relation to the psc-metrics we are going to construct.

The ideas behind the following theorem go back to Gromov-Lawson [12] and Gajer [10].

Theorem 2.2 (Walsh [28, Theorem 2.5]) Let $W$ be a smooth compact cobordism with $\partial W=M_{0} \sqcup M_{1}$. Assume that $g_{0}$ is a positive scalar curvature metric on $M_{0}$ and $(f, \mathfrak{m})$ is an admissible Morse pair on $W$. Then there is a psc-metric $\bar{g}=\bar{g}\left(g_{0}, f, \mathfrak{m}\right)$ on $W$ which extends $g_{0}$ and has a product structure near the boundary. 
Proof We will provide here only an outline and refer to Walsh [28, Theorem 2.5] for details. We begin with a few topological observations. For simplicity, we assume for
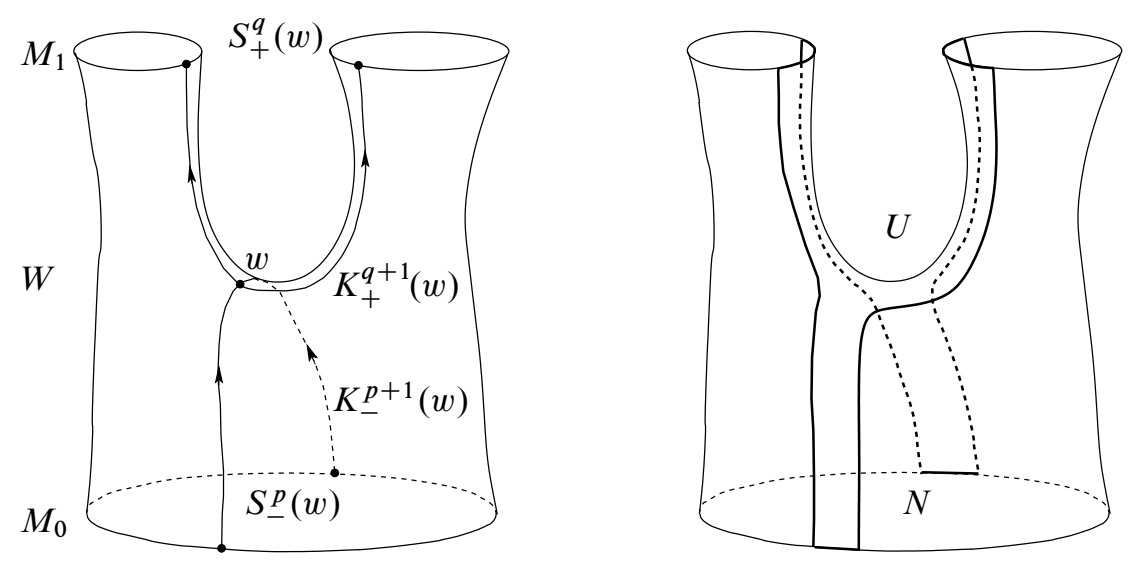

Figure 1: Trajectory disks of the critical point $w$ contained inside a disk $U$

the moment that $W$ is an elementary cobordism, that is, $f$ has a single critical point $w$ of index $p+1$. The general case is obtained by repeating the construction for each critical point. Fix a gradient like vector field for $f$. Intersecting transversely at $w$ there is a pair of trajectory disks $K_{\underline{p}}^{+1}$ and $K_{+}^{q+1}$, see Figure 1 . Here the lower $(p+1)-$ dimensional disk $K^{\underline{p}}+1$ is bounded by an embedded $p$-sphere $S \underline{p} \subset M_{0}$. It consists of the union of segments of integral curves of the gradient vector field beginning at the bounding sphere and ending at $w$. Here and below we use the compatible metric $\mathfrak{m}$ for all gradient vector fields. Similarly, $K_{+}^{q+1}$ is a $(q+1)$-dimensional disk which is bounded by an embedded $q$-sphere $S_{+}^{q} \subset M_{1}$. The spheres $S_{\underline{p}}$ and $S_{+}^{q}$ are known as trajectory spheres associated to the critical point $w$. As usual, the sphere $S_{\underline{p}}^{p} \subset M_{0}$ is embedded into $M_{0}$ together with its neighbourhood $N=S_{-}^{p} \times D^{q+1} \subset M_{0}$.

We denote by $U$ the union of all trajectories originating at the neighborhood $N$, and notice that $U$ is a disk-shaped neighbourhood of $K_{\underline{p}}^{p+1} \cup K_{+}^{q+1}$, see Figure 1. A continuous shrinking of the radius of $N$ down to zero induces a deformation retraction of $U$ onto $K^{p+1} \cup K_{+}^{q+1}$.

Now we consider the complement $W \backslash U$, which coincides with the union of all trajectories originating at $M_{0} \backslash N$. By assumption none of these trajectories have critical points. We use the normalized gradient vector field of $f$ to specify a diffeomorphism

$$
\psi: W \backslash U \rightarrow\left(M_{0} \backslash N\right) \times[0,1] .
$$


Now we construct the metric $\bar{g}$. On the region $W \backslash U$, we define the metric $\bar{g}$ to be simply $\left.g_{0}\right|_{M_{0} \backslash N}+d t^{2}$ where the $t$ coordinate comes from the embedding $\psi$ above. To extend this metric over the region $U$, we have to do more work. Notice that the

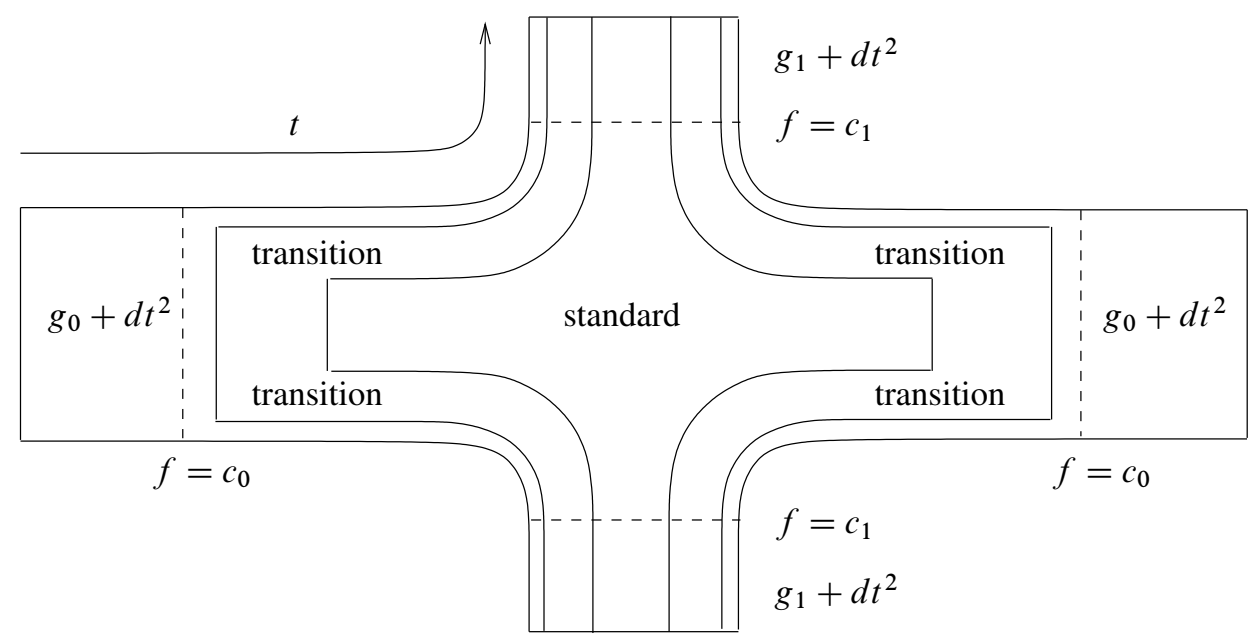

Figure 2: The metric $\bar{g}$ on the disk $U$

boundary of $U$ decomposes as

$$
\partial U=\left(S^{p} \times D^{q+1}\right) \cup\left(S^{p} \times S^{q} \times I\right) \cup\left(D^{p+1} \times S^{q}\right) .
$$

Here $S^{p} \times D^{q+1} \subset M_{0}$ is of course the tubular neighbourhood $N$ while the $D^{p+1} \times$ $S^{q} \subset M_{1}$ piece is a tubular neighbourhood of the outward trajectory sphere $S_{+}^{q} \subset M_{1}$.

Without loss of generality assume that $f(w)=\frac{1}{2}$. Let $c_{0}$ and $c_{1}$ be constants satisfying $0<c_{0}<\frac{1}{2}<c_{1}<1$. The level sets $f=c_{0}$ and $f=c_{1}$ divide $U$ into three regions:

$$
\begin{aligned}
U_{0} & =f^{-1}\left(\left[0, c_{1}\right]\right) \cap U, \\
U_{w} & =f^{-1}\left(\left[c_{0}, c_{1}\right]\right) \cap U, \\
U_{1} & =f^{-1}\left(\left[c_{1}, 1\right]\right) \cap U .
\end{aligned}
$$

The region $U_{0}$ is diffeomorphic to $N \times\left[0, c_{1}\right]$. We use again the flow to identify $U_{0}$ with $N \times\left[0, c_{1}\right]$ in a way compatible with the identification of $W \backslash U$ with $M_{0} \backslash N \times I$. Then, on $U_{0}$, we define $\bar{g}$ as the product $\left.g_{0}\right|_{N}+d t^{2}$. Moreover, we extend this metric $\left.g_{0}\right|_{N}+d t^{2}$ near the $S^{p} \times S^{q} \times I$ part of the boundary, where again $t$ is the trajectory coordinate. 
We will now define a family of particularly useful psc-metrics on the disk $D^{k}$. For a detailed discussion see Walsh [28].

Definition 2.3 Let $\delta>0$ and $\rho_{\delta}$ be a smooth function $\rho_{\delta}:(0, \infty) \rightarrow \mathbb{R}$ satisfying the following conditions:

(1) $\rho_{\delta}(t)=\delta \sin \left(\frac{t}{\delta}\right)$ when $t$ is near 0 ;

(2) $\rho_{\delta}(t)=\delta$ when $t \geq \delta \cdot \frac{\pi}{2}$;

(3) $\ddot{\rho}_{\delta}(t) \leq 0$.

Clearly such functions $\rho_{\delta}$ exists, futhermore, the space of functions satisfying the conditions (1), (2), (3) for some $\delta>0$ is convex. Let $r$ be the standard radial distance function on $\mathbb{R}^{k}$, and $d s_{k-1}^{2}$ be the standard metric on $S^{k-1}$ (of radius one). Then the metric $d r^{2}+\rho_{\delta}(r)^{2} d s_{k-1}^{2}$ on $(0, \infty) \times S^{k-1}$ is well-defned on $\mathbb{R}^{k}$. By restricting this metric to $(0, b] \times S^{k-1}$, one obtains the metric $g_{\text {tor }}^{k}(\delta)$ on $D^{k}$. This metric is defined to be a torpedo metric, see Figure 3.
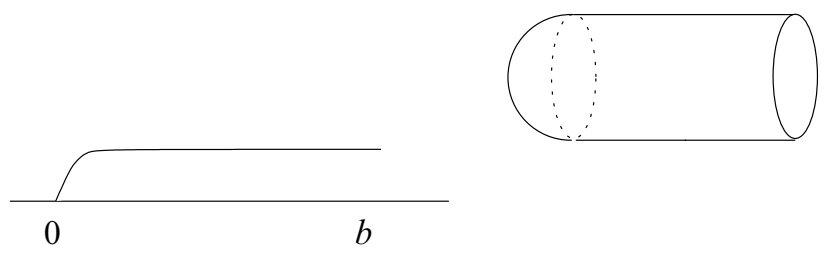

Figure 3: A torpedo function and the resulting torpedo metric

Remark It is easy to show that the above conditions (1), (2), (3) guarantee that $g_{\text {tor }}^{k}(\delta)$ has positive scalar curvature. Moreover it is $S O(k)$-symmetric and is a product with the standard metric on the $(k-1)$-sphere of radius $\delta$ near the boundary of $D^{k}$ and is the standard metric on the $k$-sphere of radius $\delta$ near the center of the disk. Also one can show that the scalar curvature of $g_{\text {tor }}^{k}(\delta)$ can be bounded below by an arbitrarily large constant by choosing $\delta$ sufficiently small.

The most delicate part of the construction, carried out carefully in [28], involves the following: Inside the region $U_{w}$, which is identified with the product $D^{p+1} \times D^{q+1}$, the metric smoothly passes into a standard product $g_{\text {tor }}^{p+1}(\epsilon)+g_{\text {tor }}^{q+1}(\delta)$ for some appropriately chosen $\epsilon, \delta>0$, globally keeping the scalar curvature positive. This is done so that the induced metric on the level set $f^{-1}\left(c_{1}\right)$, denoted $g_{1}$, is precisely the 
metric obtained by applying the Gromov-Lawson construction to $g_{0}$. Furthermore, near $f^{-1}\left(c_{1}\right)$ we have $\bar{g}=g_{1}+d t^{2}$. Finally, on $U_{1}$, which is identified with $D^{p+1} \times S^{q} \times\left[c_{1}, 1\right]$ in the usual manner, the metric $\bar{g}$ is simply the product $g_{1}+d t^{2}$. See Figure 2 for an illustration.

After the choice of the Morse coordinate diffeomorphism with $D^{p+1} \times D^{q+1}$ (and of the other parameters like $\epsilon$ and $\delta$ ), the construction is explicit and depends continuously on the given metric $g_{0}$ on $S^{p} \times D^{q+1}$.

Later on we will need the following additional facts. The next lemma is proved by Walsh [28, Section 3].

Lemma 2.4 The "initial" transition consists of an isotopy. In particular, $g_{0}$ is isotopic to a metric which, on a neighborhood diffeomorphic to $S^{p} \times D^{q+1}$ of the surgery sphere $S_{\underline{p}}^{p}$ in $M_{0}$, is $\delta^{2} d s_{p}^{2}+g_{\text {tor }}^{q+1}(\delta)$.

Lemma 2.5 The whole construction is $O(p+1) \times O(q+1)$-equivariant.

Proof By construction, the standard product of torpedo metrics is $O(p+1) \times O(q+1)-$ invariant. It is a matter of carefully going through the construction of the transition metric in [28] to check that this construction is equivariant for the obvious action of these groups. This is done by Walsh [29, Lemma 2.2].

Lemma 2.5 will be of crucial importance later, when in a non-trivial family we cannot choose globally defined Morse coordinates giving diffeomorphisms to $D^{p+1} \times D^{q+1}$ (as the bundle near the critical set is not trivial). We will construct Morse coordinates well defined up to composition with elements of $O(p+1) \times O(q+1)$. The equivariance of Lemma 2.5 then implies that our construction, which a priori depends on the choice of these coordinates, is consistent and gives rise to a smooth globally defined family of metrics.

We should emphasize that this construction can be carried out for a tubular neighbourhood $N$ of arbitrarily small radius and for $c_{0}$ and $c_{1}$ chosen arbitrarily close to $\frac{1}{2}$. Thus the region $U_{w}$, on which the metric $\bar{g}$ is not simply a product and is undergoing some kind of transition, can be made arbitrarily small with respect to the background metric $\mathfrak{m}$. As critical points of a Morse function are isolated, it follows that this construction generalizes easily to Morse functions with more than one critical point.

\subsection{Extension to families}

There is a number of ways to generalize the surgery procedure to families of manifolds. A construction relevant to our goals leads to families of Morse functions, or maps with fold singularities. We start with a local description. 
Definition 2.6 A map $F: \mathbb{R}^{k} \times \mathbb{R}^{n+1} \rightarrow \mathbb{R}^{k} \times \mathbb{R}$ is called a standard map with a fold singularitiy of index $\lambda$, if there is a $c \in \mathbb{R}$ so that $f$ is given as

$$
\begin{aligned}
\mathbb{R}^{k} \times \mathbb{R}^{n+1} & \longrightarrow \mathbb{R}^{k} \times \mathbb{R}, \\
(y, x) & \longmapsto\left(y, c-x_{1}^{2}-\cdots-x_{\lambda}^{2}+x_{\lambda+1}^{2}+\cdots+x_{n+1}^{2}\right) .
\end{aligned}
$$

Roughly speaking, the composition

$$
\mathbb{R}^{k} \times \mathbb{R}^{n+1} \stackrel{F}{\rightarrow} \mathbb{R}^{k} \times \mathbb{R} \stackrel{p_{2}}{\rightarrow} \mathbb{R}
$$

with the projection $p_{2}$ onto the second factor defines a $\mathbb{R}^{k}$-parametrized family of Morse functions of index $\lambda$ on $\mathbb{R}^{n+1}$ in standard form.

Let $W$ be a compact manifold with boundary $\partial W \neq \varnothing, \operatorname{dim} W=n+1$. We denote by $\operatorname{Diff}(W, \partial W)$ the group of all diffeomorphisms of $W$ which restrict to the identity near the boundary $\partial W$. Then we consider a smooth fiber bundle $\pi: E \rightarrow B$ with fiber $W$, where $\operatorname{dim} B=k$ and $\operatorname{dim} E=n+1+k$. The structure group of this bundle is assumed to be $\operatorname{Diff}(W, \partial W)$ and the base space $B$ to be a compact smooth manifold. Assume that the boundary $\partial W$ is split into a disjoint union: $\partial W=\partial_{0} W \sqcup \partial_{1} W$.

Let $\pi_{0}: E_{0} \rightarrow B$ and $\pi_{1}: E_{1} \rightarrow B$ be the restriction of the fiber bundle $\pi: E \rightarrow B$ to the fibers $\partial_{0} W$ and $\partial_{1} W$ respectively. Since each element of the structure group $\operatorname{Diff}(W, \partial W)$ restricts to the identity near the boundary, the fiber bundles $\pi_{0}: E_{0} \rightarrow B$ and $\pi_{1}: E_{1} \rightarrow B$ are trivialized:

$$
E_{0}=B \times \partial_{0} W \stackrel{\pi_{0}}{\longrightarrow} B, \quad E_{1}=B \times \partial_{1} W \stackrel{\pi_{1}}{\longrightarrow} B .
$$

Choose a splitting of the tangent bundle $\tau_{E}$ of the total space as $\tau_{E} \cong \pi^{*} \tau_{B} \oplus \mathcal{V}$ ert, where $\mathcal{V}$ ert is the bundle tangent to the fibers $W$, that is, choose a connection.

Definition 2.7 Let $\pi: E \rightarrow B$ be a smooth bundle as above. For each $z$ in $B$ let

$$
i_{z}: W_{z} \rightarrow E
$$

be the inclusion of the fiber $W_{z}:=\pi^{-1}(z)$. Let $F: E \rightarrow B \times I$ be a smooth map. The map $F$ is said to be an admissible family of Morse functions or admissible with fold singularities with respect to $\pi$ if it satisfies the following conditions:

(1) The diagram

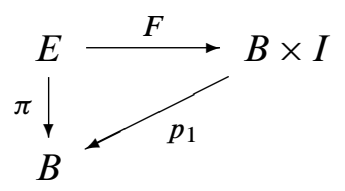

commutes. Here $p_{1}: B \times I \rightarrow B$ is projection on the first factor. 
(2) The pre-images $F^{-1}(B \times\{0\})$ and $F^{-1}(B \times\{1\})$ coincide with the submanifolds $E_{0}$ and $E_{1}$ respectively.

(3) The set $\operatorname{Cr}(F) \subset E$ of critical points of $F$ is contained in $E \backslash\left(E_{0} \cup E_{1}\right)$ and near each critical point of $F$ the bundle $\pi$ is equivalent to the trivial bundle $\mathbb{R}^{k} \times \mathbb{R}^{n+1} \stackrel{p_{1}}{\rightarrow} \mathbb{R}^{k}$ so that with respect to these coordinates on $E$ and on $B$ the map $F$ is a standard map $\mathbb{R}^{k} \times \mathbb{R}^{n+1} \rightarrow \mathbb{R}^{k} \times \mathbb{R}$ with a fold singularity as in Definition 2.6

(4) For each $z \in B$ the restriction

$$
f_{z}=\left.F\right|_{W_{z}}: W_{z} \rightarrow\{z\} \times I \stackrel{p_{2}}{\longrightarrow} I
$$

is an admissible Morse function as in Section 2.1. In particular, its critical points have indices $\leq n-2$.

We assume in addition that the smooth bundle $\pi: E \rightarrow B$ is a Riemannian submersion $\pi:\left(E, \mathfrak{m}_{E}\right) \rightarrow\left(B, \mathfrak{m}_{B}\right)$, see Besse [3]. Here we denote by $\mathfrak{m}_{E}$ and $\mathfrak{m}_{B}$ the metrics on $E$ and $B$ corresponding to the submersion $\pi$. Now let $F: E \rightarrow B \times I$ be an admissible map with fold singularities with respect to $\pi$ as in Definition 2.7. If the restriction $\mathfrak{m}_{z}$ of the submersion metric $\mathfrak{m}_{E}$ to each fiber $W_{z}, z \in B$, is compatible with the Morse function $f_{z}=\left.F\right|_{W_{z}}$, we say that the metric $\mathfrak{m}_{E}$ is compatible with the map $F$.

Proposition 2.8 Let $\pi: E \rightarrow B$ be a smooth bundle as above and $F: E \rightarrow B \times I$ be an admissible map with fold singularities with respect to $\pi$. Then the bundle $\pi: E \rightarrow B$ admits the structure of a Riemannian submersion $\pi:\left(E, \mathfrak{m}_{E}\right) \rightarrow\left(B, \mathfrak{m}_{B}\right)$ such that the metric $\mathfrak{m}_{E}$ is compatible with the map $F: E \rightarrow B \times I$.

Proof One can choose a Riemannian metric $\mathfrak{m}_{B}$ on the base $B$, and for each fiber $W_{z}$ there is a metric $\mathfrak{m}_{z}$ compatible with the Morse function $f_{z}=\left.F\right|_{W_{z}}$. Using convexity of the set of compatible metrics and the local triviality in the definition of a family of Morse functions, we can choose this family to depend continuously on $z$. Then one can choose an integrable distribution (sometimes called connection) to construct a submersion metric $\mathfrak{m}_{E}$ which is compatible with the map $F: E \rightarrow B \times I$, see [3].

Below we assume that the fiber bundle $\pi: E \rightarrow B$ is given the structure of a Riemannian submersion $\pi:\left(E, \mathfrak{m}_{E}\right) \rightarrow\left(B, \mathfrak{m}_{B}\right)$ such that the metric $\mathfrak{m}_{E}$ is compatible with the map $F: E \rightarrow B \times I$.

Consider the critical set $\operatorname{Cr}(F) \subset E$. It follows from the definitions that $\operatorname{Cr}(F)$ is a smooth $k$-dimensional submanifold in $E$, and it splits into a disjoint union of path 
components ("folds")

$$
\operatorname{Cr}(F)=\Sigma_{1} \sqcup \cdots \sqcup \Sigma_{s} .
$$

Furthemore, it follows that the restriction of the fiber projection

$$
\left.\pi\right|_{\Sigma_{j}}: \Sigma_{j} \longrightarrow B
$$

is a local diffeomorphism for each $j=1, \ldots, s$. In particular, $\left.\pi\right|_{\Sigma_{j}}$ is a covering map, and if the base $B$ is simply-connected then $\left.\pi\right|_{\Sigma_{j}}$ is a diffeomorphism onto its image.

Since the metric $\mathfrak{m}_{E}$ is a submersion metric, the structure group of the vector bundle $\mathcal{V}$ ert $\rightarrow E$ is reduced to $O(n+1)$. Futhermore, since the metrics $\mathfrak{m}_{z}$ are compatible with the Morse functions $f_{z}=\left.F\right|_{W_{z}}$, the restriction $\left.\mathcal{V} e r t\right|_{\Sigma_{j}}$ to a fold $\Sigma_{j} \subset \operatorname{Cr}(F)$ splits further orthogonally into the positive and negative eigenspaces of the Hessian of $F$. Thus the metric $\mathfrak{m}_{E}$ induces the splitting of the vector bundle

$$
\left.\mathcal{V} \operatorname{ert}\right|_{\Sigma_{j}} \cong \mathcal{V}_{e r t}^{-} \oplus \mathcal{V}_{j} r_{j}^{+}
$$

with structure group $O(p+1) \times O(q+1)$ for each $\Sigma_{j}$. Here is the main result of this section:

Theorem 2.9 Let $\pi: E \rightarrow B$ be a smooth bundle, where the fiber $W$ is a compact manifold with boundary $\partial W=M_{0} \sqcup M_{1}$, the structure group is $\operatorname{Diff}(W, \partial W)$ and the base space $B$ is a compact smooth simply connected manifold. Let $F: E \rightarrow B \times I$ be an admissible map with fold singularities with respect to $\pi$. In addition, we assume that the fiber bundle $\pi: E \rightarrow B$ is given the structure of a Riemannian submersion $\pi:\left(E, \mathfrak{m}_{E}\right) \rightarrow\left(B, \mathfrak{m}_{B}\right)$ such that the metric $\mathfrak{m}_{E}$ is compatible with the map $F: E \rightarrow$ $B \times I$. Finally, we assume that we are given a smooth map $g_{0}: B \rightarrow \mathcal{R i e m}^{+}\left(M_{0}\right)$.

Then there exists a Riemannian metric $\bar{g}=\bar{g}\left(g_{0}, F, \mathfrak{m}_{E}\right)$ on $E$ such that for each $z \in B$ the restriction $\bar{g}(z)=\left.\bar{g}\right|_{W_{z}}$ to the fiber $W_{z}=\pi^{-1}(z)$ satisfies the following properties:

(1) $\bar{g}(z)$ extends $g_{0}(z)$;

(2) $\bar{g}(z)$ is a product metric $g_{v}(z)+d t^{2}$ near $M_{v} \subset \partial W_{z}, v=0,1$;

(3) $\bar{g}(z)$ has positive scalar curvature on $W_{z}$.

Proof We assume that $B$ is path-connected. Let $\operatorname{dim} B=k, \operatorname{dim} W=n+1$. We denote, as above, $\operatorname{Cr}(F)=\Sigma_{1} \sqcup \ldots \sqcup \Sigma_{s}$, where the $\Sigma_{j}$ is a path-connected fold. For a given point $z \in B$, we denote by $f_{z}=\left.F\right|_{W_{z}}: W_{z} \rightarrow I$ the corresponding admissible Morse function.

The metric $\bar{g}$ will be constructed by a method which is quite similar to that employed in the proof of Theorem 2.2. We begin by equipping the boundary component $E_{0}$ with 
the given Riemannian metric $g_{0}$. We choose a gradient-like vector field $V$ and use the trajectory flow of $V$ to extend $\bar{g}_{0}$ as a product metric away from the folds $\operatorname{Cr}(F)$. Near the folds $\operatorname{Cr}(F)$, some modification is necessary. However, roughly speaking, the entire construction goes through in such a way that the restriction to any fiber is the construction of Theorem 2.2.

We will initially assume that $\operatorname{Cr}(F)$ has exactly one path-connected component $\Sigma$. The more general case will follow from this by iterated application of the construction. We will denote by $c$ the critical value associated with the fold $\Sigma$, that is, $p_{2} \circ F(\Sigma)=c \in I$. Let $\epsilon_{c}>0$ be small. Let $V$ denote the normalized gradient vector field associated to $F$ and $\mathfrak{m}_{E}$ which is well-defined away from the singularities of $F$. As $F$ has no other critical values, we use $V$ to specify a diffeomorphism

$$
\begin{aligned}
\phi_{0}: E_{0} \times\left[0, c-\epsilon_{c}\right] & \longrightarrow F^{-1}\left(B \times\left[0, c-\epsilon_{c}\right]\right) \\
(w, t) & \longmapsto\left(h_{w}(t)\right),
\end{aligned}
$$

where $h_{w}$ is the integral curve of $V$ beginning at $w$. In particular, $p_{2} \circ F \circ \phi_{0}$ is the projection onto $\left[0, c-\epsilon_{c}\right]$. As the bundle $\pi_{0}: E_{0} \rightarrow B$ is trivial, this gives rise to a diffeomorphism

$$
B \times M_{0} \times\left[0, c-\epsilon_{c}\right] \cong F^{-1}\left(B \times\left[0, c-\epsilon_{c}\right]\right)
$$

Let $\bar{g}_{c-\epsilon_{c}}$ denote the metric obtained on $F^{-1}\left(B \times\left[0, c-\epsilon_{c}\right]\right)$ by pulling back, via this diffeomorphism, the warped product metric $\mathfrak{m}_{B}+g_{0}+d t^{2}$. In order to extend this metric past the fold $\Sigma$, we must adapt our construction.

Our next goal is to construct a metric $\bar{g}_{c+\epsilon_{c}}$ on $F^{-1}\left(B \times\left[0, c+\epsilon_{c}\right]\right)$, so that on each fiber

$$
\pi^{-1}(y) \cap F^{-1}\left(B \times\left[0, c+\epsilon_{c}\right]\right)
$$

the induced metric has positive scalar curvature and is a product near the boundary. Fiberwise, this is precisely the situation dealt with in Theorem 2.2. However, performing this over a family of Morse critical points, we must ensure compatibility of our construction over the entire family. The main problem is that our construction depends on the choise of "Morse coordinates", that is, the diffeomorphism of a neighborhood of the critical point to $D^{p+1} \times D^{q+1}$. Because of the non-triviality of the bundle, a global choice of this kind is in general not possible. We will normalize the situation in such a way that we choose diffeomorphisms up to precomposition with elements of $O(p+1) \times O(q+1)$ (in some sense a suitable reduction of the structure group). We then use Lemma 2.5, that the construction employed is equivariant for this smaller group $O(p+1) \times O(q+1)$. 
Our strategy actually is to use the fiberwise exponential map for $\mathfrak{m}_{z}$ at the critical set as Morse coordinates. Because of the canonical splitting

$$
\mathcal{V} \text { ert }\left.\right|_{\Sigma}=\mathcal{V}_{e r t}{ }^{-} \oplus \mathcal{V}_{e r t}{ }^{+}
$$

with structure group $O(p+1) \times O(q+1)$ this gives coordinates which are well defined up to an action of $O(p+1) \times O(q+1)$ (the choice of orthonormal bases in Vert $^{+}$ and $\left.\mathcal{V e r t}^{-}\right)$. However, these coordinates are not Morse coordinates for $F$. That the metrics $\mathfrak{m}_{z}$ are compatible with the Morse function $f_{z}$ only means that this is the case infinitesimally. We will therefore deform the given Morse function $F$ to a new Morse function $F_{1}$ for which our coordinates are Morse coordinates.

We denote by $D \operatorname{Vert}_{\Sigma}$ the corresponding disk bundle of radius $\delta$ with respect to the background metric $\mathfrak{m}_{E}$. For each $w \in \Sigma$, we denote by $D_{w}\left(\mathcal{V}\right.$ ert $\left.t_{\Sigma}\right)$ the fiber of this bundle. If $\delta$ is sufficiently small, the fiberwise exponential map (and local orthonormal bases for $\mathcal{V e r t}^{+}$and $\mathcal{V}$ ert ${ }^{-}$) define coordinates $D^{p+1} \times D^{q+1}$ for neighborhoods of the critical point in each fiber. We use the exponential map to pull back all structures to $D^{p+1} \times D^{q+1}$ and, abusing notation, denote them in the old way. In particular, the function $F$ is defined on $D^{p+1} \times D^{q+1}$.

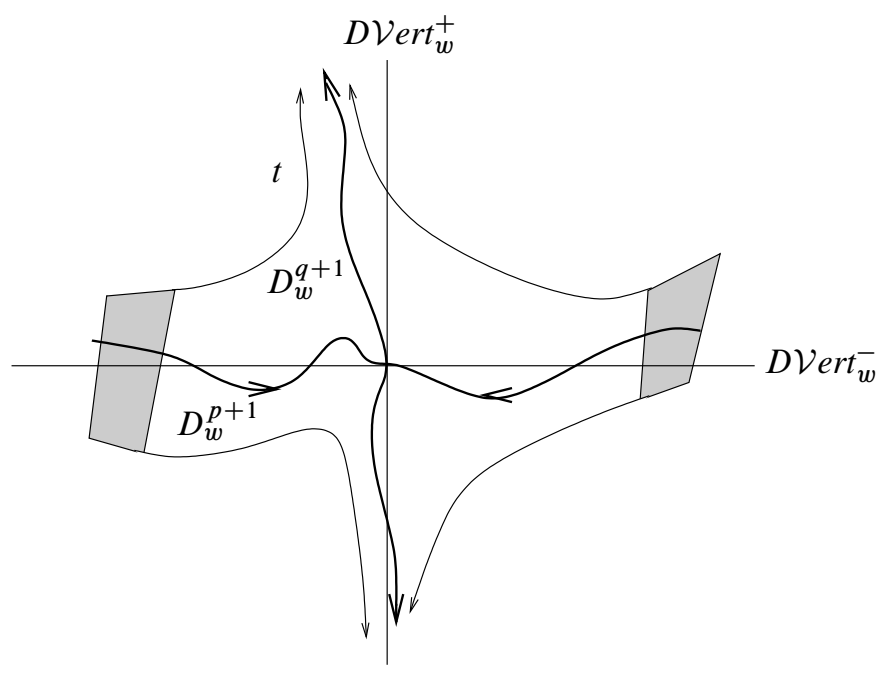

Figure 4: The images of the trajectory disks $D_{w}^{p+1}$ and $D_{w}^{q+1}$ in $D_{w} \mathcal{V} \operatorname{ert}(\Sigma)$ after application of the inverse exponential map

Let $\rho$ and $r$ denote the distance to the origin in $D^{p+1}$ and $D^{q+1}$, respectively. Then $\rho^{2}$ and $r^{2}$ are smooth functions on the image under the fiberwise exponential map 
of $D\left(\mathcal{V e r t}_{\Sigma}\right)$. Moreover, define $F_{\text {std }}: D \mathcal{V}$ ert $t_{\Sigma} \rightarrow \mathbb{R}$ by $F_{\text {std }}:=c-\rho^{2}+r^{2}$. The compatibility condition on $F$ and the Taylor expansion theorem imply that $F-F_{\text {std }}=$ $O\left({\sqrt{r^{2}+\rho^{2}}}^{3}\right)$, that is, $F-F_{\text {std }}$ is cubic in the $\mathfrak{m}_{z}$-distance to the origin.

Choose a sufficiently small $\alpha>0$ and a smooth cutoff function $\phi_{\alpha}: \mathbb{R} \rightarrow[0,1]$ with

(1) $\phi_{\alpha}(s)=1$ for $s<\alpha$

(2) $\phi_{\alpha}(s)=0$ for $s>2 \alpha$

(3) $\left|\phi^{\prime}(s)\right| \leq 10 / \alpha \quad \forall s \in \mathbb{R}$.

Then $F_{t}:=F_{\text {std }}+\left(1-t \phi_{\alpha}\left(\sqrt{\rho^{2}+r^{2}}\right)\right)\left(F-F_{\text {std }}\right)$ provides a homotopy between $F=F_{0}$ and $F_{1}$ of families of Morse functions with the following properties:

(1) $\operatorname{Cr}\left(F_{t}\right)=\operatorname{Cr}(F) \forall t \in[0,1]$;

(2) $F_{t}$ coincides with $F$ outside of a tubular neigbourhood of $\Sigma \forall t \in[0,1]$;

(3) $\quad F_{1}=F_{\text {std }}$ on a sufficiently small neighbourhood of the fold $\Sigma$ in DVert $\Sigma$.

The second and the third condition are evident. For the first, we have to check that we did not introduce new critical points. Now the gradient of $F_{\text {std }}$ is easily calculated and its norm at $x$ is equal to the norm of $x$. On the other hand, the gradient of

$$
\left(1-t \phi\left(\sqrt{r^{2}+\rho^{2}}\right)\right)\left(F-F_{\text {std }}\right)
$$

has two summands:

(1) The first one is $\left(1-t \phi\left(\sqrt{r^{2}+\rho^{2}}\right)\right) \nabla\left(F-F^{\prime}\right)$, where $(1-t \phi)$ is bounded and $\nabla\left(F-F^{\prime}\right)$ is quadratic in the distance to the origin (as $F-F^{\prime}$ has a Taylor expansion which starts with cubic terms).

(2) The second one is $t \phi^{\prime}\left(\sqrt{r^{2}+\rho^{2}}\right) \nabla\left(\sqrt{r^{2}+\rho^{2}}\right)\left(F-F^{\prime}\right)$. This vanishes identically if $r^{2}+\rho^{2} \leq \alpha^{2}$, and is bounded by $10\left(F-F^{\prime}\right) / \alpha 0 \leq 10\left(F-F^{\prime}\right) / \sqrt{r^{2}+\rho^{2}}$ if $r^{2}+\rho^{2} \geq \alpha^{2}$ (here we use that the gradient of the distance to the origin $\sqrt{r^{2}+\rho^{2}}$ has norm 1$)$. Since $F-F^{\prime}$ is cubic in $\sqrt{r^{2}+\rho^{2}}$, the whole expression is quadratic.

It follows that, if $\alpha$ is chosen small enough (there is a uniform bound because we deal with a compact family, so we find uniform bounds for the implicit constants in the above estimates), the gradient of $F_{t}$ vanishes exactly at the origin. Near the origin, by the choice of $\phi, F_{t}=t F_{\text {std }}+(1-t) F$. Because the Hessians of $F$ and of $F_{\text {std }}$ are identical at the origin, the Hessian of $F_{t}$ also coincides with the Hessian of $F_{\text {std }}$, in particular $F_{t}$ is a family of Morse functions. To find the required local Morse coordinates, we can invoke Igusa's [16, Theorem 1.4]. 
Thus we can assume that the map $F$ is standard near the fold $\Sigma$, that is, $F=F_{1}$ in the first place, and from now on we will do so.

Now, via the fiberwise exponential map for $\mathfrak{m}_{\pi(w)}$, for each $w \in \Sigma$ we can specify a neighbourhood $U_{w} \subset \pi^{-1}(\pi(w))$ containing the point $w$ and of the type described in the proof of Theorem 2.2. In Figures 4 and 5, the image of this region under the inverse exponential map, before and after the above adjustment of $F$, is shown. For each $w \in \Sigma$, replace the fiber $D_{w} \mathcal{V e r t}_{\Sigma}$ with the image under the inverse exponential map of $U_{w}$. Abusing notation we will retain the name $D \mathcal{V}$ ert $t_{\Sigma}$ for this bundle, the fibers of which should be thought of as the cross-shaped region described in Figure 5. The structure group of this bundle is still $O(p+1) \times O(q+1)$. The metric induced

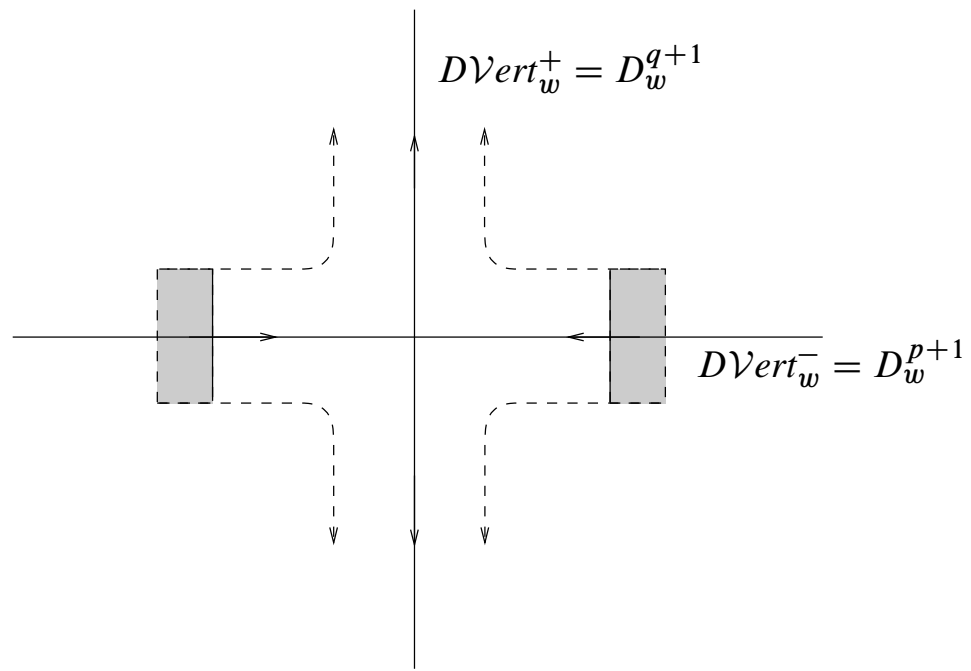

Figure 5: The shaded region denotes the region of the fiber $D_{w} \mathcal{V} \operatorname{ert}(F)$ on which the induced metric is defined.

by $\bar{g}_{c-\epsilon_{c}}$ is defined on a subbundle with fibers diffeomorphic to $S^{p} \times D^{q+1} \times I$, see Figure 5. On each fiber we now perform the construction from Theorem 2.2. The fact that we adjusted $F$ to make the trajectories standard on the fiber disk guarantees consistency of the construction. On each fiber there is a splitting into positive and negative eigenspaces over which we will perform our construction. We must however, choose a pair of orthonormal bases for the negative and positive eigenspaces of that fiber in order to appropriately identify the fiber with Euclidean space. In order to guarantee consistency we must ensure that our construction is independent of these choices. But this follows from Lemma 2.5. 
Extending the metric fiberwise in the manner of Theorem 2.2 and pulling back via the exponential map, gives a smooth family of fiber metrics, which, with respect to some integrable distribution $H$ and the base metric $\mathfrak{m}_{B}$, combine to the desired submersion metric on $F^{-1}\left(B \times\left[0, c+\delta_{c}\right]\right)$.

Remark With some little extra care it should be possible to remove the condition that $B$ is simply connected in Theorem 2.9. However, we are only interested in the case $B=S^{n}$ with $n>1$ so that, for our purpose, we can stick to the simpler version as stated.

\section{Metrics of positive scalar curvature on Hatcher's examples}

The work of Goette [11, Section 5.b] shows that Hatcher's examples can be given the structure which is described in Definition 2.7. The construction of the Hatcher bundles $D^{n} \rightarrow E \rightarrow S^{k}$ is explained in some detail in [11] and will not be repeated here. Most important for our discussion is the fact that each of these bundles comes with an admissible family $F$ of Morse functions as indicated in the following commutative diagram:

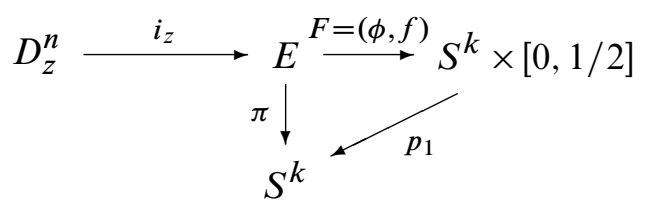

We follow the description given in [11]. Each $f_{z}:=\left.f\right|_{E_{z}}: D_{z}^{n} \rightarrow[0,1 / 2]$ has three critical points $p_{z}^{(0)}, p_{z}^{(1)}$ and $p_{z}^{(2)}$. In particular, the points $p_{z}^{(0)}$ form a unique fiberwise minimum of the Morse functions $f_{z}$ with value 0 , and $F^{-1}\left(S^{k} \times\{0\}\right)$ has a neighborhood $F^{-1}\left(S^{k} \times[0,1 / 8]\right)$ which (as a smooth bundle) is diffeomorphic to $D^{n} \times S^{k}$. Near the value $1 / 2$, the inverse image $F^{-1}\left(S^{k} \times\{1 / 2\}\right)$ has a neighborhood diffeomorphic to $\left(S^{n-1} \times I\right) \times S^{k}$. We now consider the upside-down copy of the bundle (8):

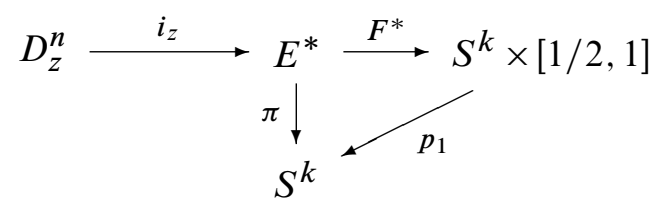

Here $E^{*}:=E$ and $F^{*}(e):=\left(\pi(e), 1-f_{\pi(e)}(e)\right)$, that is, $f_{z}^{*}=1-f_{z}$, where we write $F=(\phi, f)$. It follows that each $f_{z}^{*}: D^{n} \rightarrow[1 / 2,1]$ has three critical points $p_{z}^{(* 0)}$, $p_{z}^{(* 1)}$ and $p_{z}^{(* 2)}$. In particular, the points $p_{z}^{(* 0)}$ form a unique fiberwise maximum 
of the Morse functions $f_{z}^{*}$ with value 1 , and $\left(F^{*}\right)^{-1}\left(S^{k} \times\{0\}\right)$ has a neighborhood $\left(F^{*}\right)-1\left(S^{k} \times[7 / 8,1]\right)$ which (as a smooth bundle) is again diffeomorphic to $D^{n} \times S^{k}$. Near the value $1 / 2$, the inverse image $\left(F^{*}\right)^{-1}\left(S^{k} \times\{1 / 2\}\right)$ again has a neighborhood diffeomorphic to $\left(S^{n-1} \times I\right) \times S^{k}$.

By cutting out the neighborhood $D^{n} \times S^{k}$ of the fiberwise minima of $F$, we obtain a smooth bundle

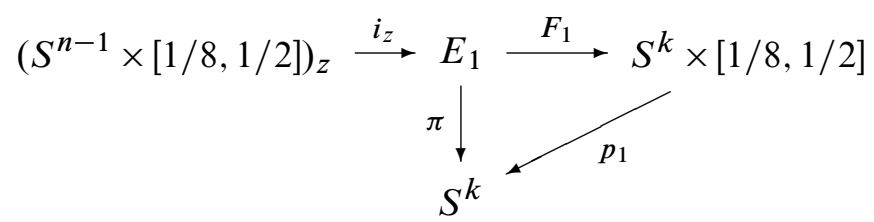

where $E_{1}:=E \backslash F^{-1}\left(S^{k} \times[0,1 / 8)\right), F_{1}:=\left.F\right|_{E_{1}}$, and the spheres $S^{n-1}$ in the product

$$
S^{n-1} \times S^{k}=F^{-1}\left(S^{k} \times\{1 / 8\}\right)
$$

are given the standard metric $g_{0}$ of fixed (but arbitrary) radius $b$ independent of $z \in S^{k}$. The bundle (10) satisfies all the assumptions of Theorem 2.9, and we obtain psc-metrics $\bar{g}_{z}$ on each fiber $\left(S^{n-1} \times[1 / 8,1 / 2]\right)_{z}$ with a product-metric near the boundary. In particular, this gives a family of metrics $\left(g_{1}\right)_{z}$ on the spheres $S_{z}^{n-1} \times\{1 / 2\}$.

Now we apply the same construction to the upside-down copy $E^{*}$ to obtain a smooth bundle $E_{1}^{*}$ with fibers $\left(S^{n-1} \times[1 / 2,7 / 8]\right)_{z}$. To make sure that the metrics match, we set $\bar{g}_{z}^{*}:=\bar{g}_{z}$, that is, we use the same metric upside-down.

Because our construction provides metrics which are products near the boundary, we can glue together the bundles $E_{1}$ and $E_{1}^{*}$ to form a bundle $\widetilde{E} \rightarrow S^{k}$ with fiber $\left(S^{n-1} \times[1 / 8,7 / 8]\right)_{z}$ together with a smooth family of psc-metrics. We notice that the restriction of the bundle $\widetilde{E}$ to the boundaries

$$
\left(S^{n-1} \times\{1 / 8,7 / 8\}\right)_{z}=\partial\left(S^{n-1} \times[1 / 8,7 / 8]\right)_{z}
$$

is trivial by construction, and the spheres $S^{n-1} \times\{1 / 8,7 / 8\}$ are given the standard metric independent of the fiber. Thus we can glue the fiberwise caps $\left(D_{0}^{n} \sqcup D_{1}^{n}\right)_{z}$ to the bundle $\widetilde{E} \rightarrow S^{k}$ by identifying

$$
\begin{aligned}
& \left(D_{0}^{n}\right)_{z} \supset S_{z}^{n-1}=\left(S^{n-1} \times\{1 / 8\}\right)_{z}, \\
& \left(D_{1}^{n}\right)_{z} \supset S_{z}^{n-1}=\left(S^{n-1} \times\{7 / 8\}\right)_{z} .
\end{aligned}
$$

Then we define the torpedo metrics $g_{\text {tor }}(r)$ on the disks $\left(D_{0}^{n}\right)_{z}$ and $\left(D_{0}^{n}\right)_{z}$ such that they match the chosen standard metric of radius $b$ on the boundary spheres. We denote 
the resulting metric on the fiber sphere $S_{z}^{n}$ by $\bar{g}_{z}$. Let $\bar{E} \rightarrow S^{k}$ be the resulting fiber bundle with fiber $S^{n}$.

Let us investigate what we have achieved: for each $z \in S^{k}$ we get a psc-metric on the fiber $S_{z}^{n}$ over $z$. This is a manifold diffeomorphic to $S^{n}$, but not with a given diffeomorphism. Hence this metric defines a point in the moduli space of pcs-metrics on $S^{n}$. Finally, there is a base point $z_{0} \in S^{n}$ together with a fixed neighborhood on which all these diffeomorphisms restrict to the identity. This implies that in fact we get an element in $\pi_{k} \mathcal{M}_{x_{0}}^{+}\left(S^{n}\right)$. The map

$$
\iota: \mathcal{M}_{x_{0}}^{+}\left(S^{n}\right) \longrightarrow \mathcal{M}_{x_{0}}\left(S^{n}\right)=B \operatorname{Diff}_{x_{0}}\left(S^{n}\right)
$$

forgets the fiberwise Riemannian metrics and just remembers the structure of $\bar{E}$ as a smooth bundle. Because for odd $n$ the generators of $\pi_{k} \mathcal{M}_{x_{0}}^{+}\left(S^{n}\right) \otimes \mathbb{Q}$ can in a stable range be represented by classifying maps of Hatcher bundles (see Bökstedt [4], Igusa [16] and Goette [11]) we have proved our first main result, Theorem 1.5.

To prove our second main result, Theorem 1.6, for a general manifold $M$, we use the above fiber bundles to form non-trivial bundles by taking a fiberwise connected sum $M \# S^{n}$.

Let $M$ be a smooth manifold with a base point $x_{0}$. We assume that $M$ is equipped with a psc-metric $h$. We fix a disk $D_{0}^{n} \subset M$ of small radius centered at $x_{0}$. We may always deform the metric $h$ near $x_{0}$ such that its restriction on $D_{0}^{n}$ is a torpedo metric $g_{\text {tor }}(r)$ (use, for example, Lemma 2.4 and thinking of the given disk as one half of a tubular neighborhood of an embedded $S^{0}$ ). Thus we will assume that the metric $h$ already has this property.

On the other hand, we consider the bundle $\bar{E} \rightarrow S^{k}$ with with psc-metrics $\bar{g}_{z}$ on the fibers $S_{z}^{n}, z \in S^{k}$, as constructed before. We notice that the metrics $\bar{g}_{z}$ are chosen in such way that their restrictions to the disks $\left(D_{0}^{n}\right)_{z}$ and $\left(D_{1}^{n}\right)_{z}$ are torpedo metrics (with chosen parameter). Let

$$
\widetilde{D}_{z}^{n}=S_{z}^{n} \backslash\left(D_{1}^{n}\right)_{z}
$$

This is a disk together with the metric $\widetilde{g}_{z}=\left.\bar{g}_{z}\right|_{\widetilde{D}_{z}^{n}}$ which is a product-metric $g_{0}+d t^{2}$ near the boundary $S_{z}^{n-1} \subset \widetilde{D}_{z}^{n}$. Now for each $z \in S^{k}$ we define the Riemannian manifold

$$
M_{z}=M \#\left(S^{n}\right)_{z}=\left(M \backslash D_{0}\right) \cup_{S_{z}^{n-1}} \widetilde{D}_{z}^{n}
$$

equipped with the metric $\tilde{h}_{z}$ so that

$$
\left.\tilde{h}_{z}\right|_{M \backslash D_{0}}=\left.h\right|_{M \backslash D_{0}},\left.\quad \tilde{h}_{z}\right|_{D_{z}^{n}}=\tilde{g}_{z} .
$$


This defines a smooth fiber bundle

$$
\widetilde{E}=\left(M \times S^{k}\right) \# E \longrightarrow S^{k},
$$

where $\left(M \times S^{k}\right) \# E$ is the total space of the fiber-wise connected sum as we just described. It follows from the additivity property (see Igusa [18, Section 3.1]) that the higher Franz-Reidemeister torsion of the fiber bundle $\widetilde{E} \rightarrow S^{k}$ is a non-zero class in $H^{k}\left(S^{k} ; \mathbb{Q}\right)$. This implies that the classifying map

$$
S^{k} \rightarrow B \operatorname{Diff}_{x_{0}}(M)=\mathcal{M}_{x_{0}}(M)
$$

of this bundle defines a non-zero element in $\pi_{k}\left(\mathcal{M}_{x_{0}}(M) ;[\tilde{h}]\right)$. Since we have constructed psc-metrics on the fibers $M_{z}$, this non-zero element can be lifted to the group $\pi_{k}\left(\mathcal{M}_{x_{0}}^{+}(M),[\tilde{h}]\right)$. This finishes the proof of Theorem 1.6.

\section{Homotopy type of the usual psc-moduli space}

In this section, we show that for a suitable choice of $M$ as in Theorem 1.6, the map $\mathcal{M}_{x_{0}}^{+}(M) \rightarrow \mathcal{M}^{+}(M)$ is non-trivial on $\pi_{k}$.

For a closed smooth manifold $M$ let $A_{H}(M)$ be the image of the canonical map $\operatorname{Diff}(M) \rightarrow \operatorname{Aut}\left(H_{*}(M ; \mathbb{Q})\right)$.

Lemma 4.1 For any $N \geq 0$ there is a closed smooth orientable manifold $M$ of dimension $n$ with the following properties:

(1) $n$ is odd and $n \geq N$.

(2) $M$ carries a psc-metric.

(3) Each $S^{1}$-action on $M$ is trivial.

(4) $A_{H}(M)$ is finite.

(5) Each diffeomorphism of $M$ is orientation preserving.

Before we explain the construction of $M$, we show how Theorem 1.7 follows.

Let $d>0$ be given and choose $N$ so that Theorem 1.5 holds for all $n \geq N$ and all $k=4 q \leq d$. For $k=4 q \leq d$ we consider the fibration

$$
M \rightarrow E \rightarrow S^{k}
$$

constructed at the end of Section 3. By construction this fibration is classified by a map $f: S^{k} \rightarrow B G$, where $G:=\operatorname{Torr}(M) \cap \operatorname{Diff}_{x_{0}}(M)$. Because the higher FranzReidemeister torsion of this bundle is a non-zero element in $H^{k}\left(S^{k} ; \mathbb{Q}\right)$, the fundamental class of $S^{k}$ is mapped to a non-zero element in $H_{k}(B G ; \mathbb{Q})$ and then further to a non-zero element $c \in H_{k}(B \operatorname{Torr}(M) ; \mathbb{Q})$. 
Let $\phi: M \rightarrow M$ be a diffeomorphism. Then $\phi$ is orientiation preserving by assumption. Because $\operatorname{Torr}(M)$ is normal in $\operatorname{Diff}(M)$, the map $\phi$ induces a map

$$
\bar{\phi}: B \operatorname{Torr}(M) \rightarrow B \operatorname{Torr}(M),
$$

where we think of $B \operatorname{Torr}(M)$ as $E \operatorname{Diff}(M) / \operatorname{Torr}(M)$.

Lemma 4.2 $\bar{\phi}_{*}(c)=c$.

Proof The map $\bar{\phi}$ is induced my the homomorphism $\operatorname{Torr}(M) \rightarrow \operatorname{Torr}(M)$ given by conjugation with $\phi$. By construction, the bundle $E$ is classified by a map $S^{k} \rightarrow$ $B \operatorname{Diff}_{x_{0}}(M, M-D)$ where $D \subset M$ is a small embedded disc around the base point $x_{0} \in M$. Note that $\operatorname{Diff}_{x_{0}}(M, M-D)$ can be regarded as a subgroup of $\operatorname{Torr}(M)$. The map $\phi$ is isotopic to a diffeomorphism fixing $D$. Conjugation by this element induces the identity homomorphism on the subgroup $\operatorname{Diff}_{x_{0}}(M, M-D) \subset \operatorname{Torr}(M)$.

We conclude that the finite group $A_{H}(M)=\operatorname{Diff}(M) / \operatorname{Torr}(M)$ acts freely on the space $E \operatorname{Diff}(M) / \operatorname{Torr}(M)=B \operatorname{Torr}(M)$ with quotient $E \operatorname{Diff}(M) / \operatorname{Diff}(M)=$ $B \operatorname{Diff}(M)$ and fixes $c \in H_{*}(B \operatorname{Torr}(M) ; \mathbb{Q})$. A transfer argument implies that $c$ is mapped to a nonzero class in $H_{*}(B \operatorname{Diff}(M) ; \mathbb{Q})$ under the canonical map $B \operatorname{Torr}(M) \rightarrow B \operatorname{Diff}(M)$.

Theorem 1.7 now follows from the observation that this class lies in the image of the Hurewicz map, from the commutativity of the diagram

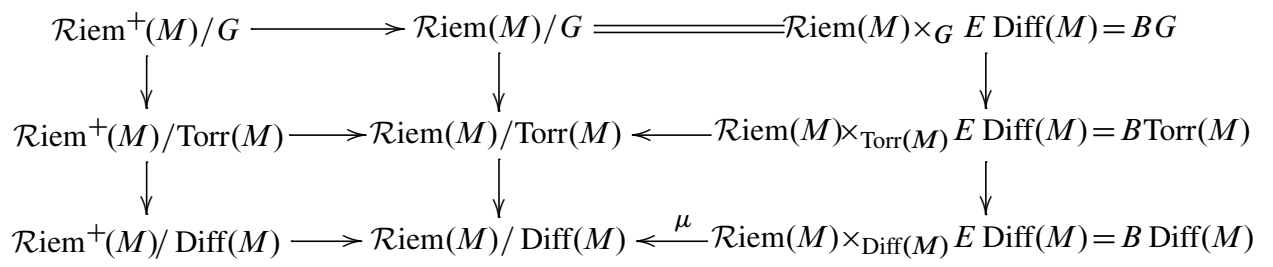

and from the following lemma.

Lemma 4.3 Assume that $\gamma \in \pi_{k}(B \operatorname{Diff}(M))$ is not in the kernel of the Hurewicz map

$$
\pi_{k}(B \operatorname{Diff}(M)) \rightarrow H_{k}(B \operatorname{Diff}(M) ; \mathbb{Q}) .
$$

Then the canonical map

$$
\mu: B \operatorname{Diff}(M)=\mathcal{R} \operatorname{iem}(M) \times_{\operatorname{Diff}(M)} E \operatorname{Diff}(M) \rightarrow \mathcal{R i e m}(M) / \operatorname{Diff}(M),
$$

sends $\gamma$ to a non-zero element in $\pi_{k}(\mathcal{R} \operatorname{iem}(M) / \operatorname{Diff}(M))$. 
Proof For $[g] \in \mathcal{R} \operatorname{iem}(M) / \operatorname{Diff}(M)$ the preimage $\mu^{-1}([g])=(g \cdot \operatorname{Diff}(M)) \times_{\operatorname{Diff}(M)}$ $E \operatorname{Diff}(M)$ is homeomorphic to $B\left(\operatorname{Diff}(M)_{g}\right)$, where $\operatorname{Diff}(M)_{g}$ is the isotropy group of $g \in \mathcal{R i e m}(M)$. Furthermore, by the existence of a local slice through $g$ for the action of $\operatorname{Diff}(M)$ on $\mathcal{R} \operatorname{iem}(M)$, which can be assumed to be $\operatorname{Diff}(M)_{g}$-linear (see, for example, Bourguignon [6, Section II.13.]), each neighbourhood of $[g] \in$ $\mathcal{R i e m}(M) / \operatorname{Diff}(M)$ contains an open neighbourhood $U$ so that $\mu^{-1}(U)$ retracts to $\mu^{-1}([g])$. In particular, the Leray sheaf $\mathcal{H}^{*}(\mu)$ for $\mu$, see Bredon [7, IV.4], is constant and equal to $\mathbb{Q}$ in degree 0 and equal to 0 in all other degrees. Here we use the Myers-Steenrod theorem (see Myers [23]) which says that $\operatorname{Diff}(M)_{g}$ is a compact Lie group and hence finite as $S^{1}$ can act only trivially on $M$. This implies that the reduced sheaf theoretic cohomology $\tilde{H}_{s h}^{*}\left(B \operatorname{Diff}(M)_{g} ; \mathbb{Q}\right)=0$ for all $g \in \mathcal{R} \operatorname{iem}(M)$ by the usual transfer argument [7, II.19] for sheaf theoretic cohomology.

We conclude that the cohomological Leray spectral sequence (see, for example, [7, IV.6])

$$
E_{2}^{p, q}=H_{s h}^{p}\left(\mathcal{R i e m}(M) / \operatorname{Diff}(M) ; \mathcal{H}^{q}(\mu)\right) \Rightarrow H_{s h}^{p+q}(B \operatorname{Diff}(M) ; \mathbb{Q})
$$

collapses at the $E_{2}$-level. From this it follows that the map $\mu$ induces an isomorphism in sheaf theoretic cohomology with rational coefficients.

In order to derive the statement of the lemma, note that up to homotopy equivalence the space $B \operatorname{Diff}(M)$ can be assumed to be a paracompact Fréchet manifold (see Kriegl and Michor [19, Section 44.21]), in particular to be locally contractible. This and the homotopy invariance of sheaf theoretic cohomology [7, Theorem II.11.12] imply by [7, Theorem III.1.1] that there is a canonical isomorphism

$$
H_{s h}^{*}(B \operatorname{Diff}(M) ; \mathbb{Q}) \cong H_{\text {sing }}^{*}(B \operatorname{Diff}(M) ; \mathbb{Q})
$$

of sheaf theoretic and singular cohomology.

Let $\gamma$ be represented by a map $S^{k} \rightarrow B \operatorname{Diff}(M)$ and consider the composition

$$
S^{k} \rightarrow B \operatorname{Diff}(M) \rightarrow \mathcal{R i e m}(M) / \operatorname{Diff}(M) .
$$

We have shown above that there is a class in $H_{s h}^{k}(\mathcal{R i e m}(M) / \operatorname{Diff}(M) ; \mathbb{Q})$ whose pullback under this composition evaluates non-zero on the singular fundamental class of $S^{k}$ (after identifying $H_{s h}^{k}\left(S^{k} ; \mathbb{Q}\right)=H_{\text {sing }}^{k}\left(S^{k} ; \mathbb{Q}\right)$ ). This implies that this composition cannot be homotopic to a constant map.

It remains to construct the manifold $M$ in Lemma 4.1.

Let $n \geq 3$ be a natural number. According to Mostow rigidity the isometry group of a closed hyperbolic $n$-manifold $M$ is isomorphic to the outer outomorphism group 
$\operatorname{Out}\left(\pi_{1}(M)\right)$. In Belolipetsky and Lubotzky [2, Theorem 1.1] a closed hyperbolic $n$-manifold $M^{n}$ with trivial isometry group is constructed. In the notation of [2], $M^{n}$ is defined as a quotient $\mathbb{H}^{n} / B$ of hyperbolic $n$-space by a discrete subgroup of Isom $\left(\mathbb{H}^{n}\right)$ which, according to [2, Section 2.3 and Remark 6.3], can be assumed to consist only of orientation preserving isometries of $\mathbb{H}^{n}$. In particular, we can assume that $M^{n}$ is orientable. Summarizing, we have

Lemma 4.4 For each $n \geq 3$, there is an orientable closed hyperbolic (hence aspherical) $n-$ manifold $B^{n}$ so that $\operatorname{Out}\left(\pi_{1}\left(B^{n}\right)\right)=1$.

Next, let $k \geq 2$ be a natural number. We construct an orientable $4 k$-dimensional manifold $N$ as follows.

Recall the Moore space $M(\mathbb{Z} / 2,2)=S^{2} \cup_{\phi} D^{3}$ where $\phi: \partial D^{3} \rightarrow S^{2}$ is of degree 2 . Its reduced integral homology is concentrated in degree 2 and isomorphic to $\mathbb{Z} / 2$. Let $S^{2} \rightarrow B \mathrm{SO}(3 k)$ represent a generator of $\pi_{2}(B \mathrm{SO}(3 k))=\mathbb{Z} / 2$. This map can be extended to a map $M(\mathbb{Z} / 2,2) \rightarrow B \mathrm{SO}(3 k)$ which then induces an isomorphism $H^{2}(B \mathrm{SO}(3 k) ; \mathbb{Z} / 2) \cong H^{2}(M(\mathbb{Z} / 2,2) ; \mathbb{Z} / 2)$ of groups that are isomorphic to $\mathbb{Z} / 2$. By pulling back the universal bundle over $B \mathrm{SO}(3 k)$ we obtain a Euclidean vector bundle $X \rightarrow M(\mathbb{Z} / 2,2)$ of rank $3 k$ which is orientable, but not spin. At this point we note that the generator of $H^{2}(B \mathrm{SO}(3 k) ; \mathbb{Z} / 2)$ is the second Stiefel-Whitney class of the universal bundle over $B \mathrm{SO}(3 k)$.

In this discussion we can replace $M(\mathbb{Z} / 2,2)$ by a homotopy equivalent finite $3-$ dimensional simplicial complex, which we denote by the same symbol. If $k$ is chosen large enough then $M(\mathbb{Z} / 2,2)$ can be embedded as a subcomplex into $\mathbb{R}^{k+1}$. We consider a regular neighbourhood $R \subset \mathbb{R}^{k+1}$ of this subcomplex. This is an compact oriented submanifold of $\mathbb{R}^{k+1}$ with boundary which contains $M(\mathbb{Z} / 2,2)$ as a deformation retract. By construction $\partial R$ is an oriented closed smooth manifold of dimension $k$. Furthermore, because $R$ has the rational homology of a point, Poincaré duality and the long exact homology sequence for the pair $(R, \partial R)$ show that $\partial R$ is a rational homology sphere. Let $E \rightarrow \partial R$ be the restriction of the pull back over $R$ of the vector bundle $X \rightarrow M(\mathbb{Z} / 2,2)$. If $k$ is chosen large enough, then $H^{2}(R ; \mathbb{Z} / 2) \rightarrow H^{2}(\partial R ; \mathbb{Z} / 2)$ is an isomorphism and hence $E$ is not spin.

Let $D E$ be the disc bundle of $E$ and let $P$ be the oriented double of $D E$. The manifold $P$ is the total space of an oriented $S^{3 k}$ bundle over $\partial R$ with vanishing Euler class (the latter for dimension reasons). Hence the rational homology of $P$ is concentrated in degrees $0, k, 3 k$ and $4 k$ and isomorphic to $\mathbb{Q}$ in these degrees. Furthermore, the manifold $P$ is orientable, but not spin. The latter holds, because the tanget bundle of 
$D E$ restricted to $\partial R$ splits as a direct sum $T(\partial R) \oplus E$ and the bundle $T(\partial R)$ is stably trivial, since it becomes trivial after adding a trivial real bundle of rank 1 .

Because $P$ is simply connected by construction, the Hurewicz theorem modulo the Serre class of finite abelian groups shows that $P$ has finite homotopy groups up to degree $k-1$.

If we additionally assume that $k$ is odd, then the only possibly non-zero Pontrijagin class of $P$ lives in degree $4 k$, hence the $\hat{A}$-genus of $P$ is a multiple of the signature of $P$ and thus equal to 0 .

There is a $4 k$-dimensional oriented closed smooth manifold $Q$, given by a Milnor $E_{8}-$ plumbing construction (see Milnor and Kervaire [22]), which is $(2 k-1)-$ connected and whose intersection form on $H^{2 k}(Q ; \mathbb{Z})$ is a direct sum of copies of the positive definite lattice $E_{8}$, hence itself a positive definite lattice. In particular, the signature of $M$ is non-zero. The first non-zero Pontrijagin class is $p_{k}(Q) \in H^{4 k}(Q ; \mathbb{Z})$, which is non-zero by the signature theorem. In particular the $\hat{A}$-genus of $Q$ is nonzero.

For later use we recall that positive definite lattices have finite automorphism groups: Given such a lattice $E$ choose a bounded ball $D$ around 0 which contains a set of generators. Because $E$ is finitely generated and positive definite, $D$ is finite. Now observe that each automorphism $E$ permutes the points in $D$ and is uniquely determined by this permutation.

We finally define the oriented manifold $N^{4 k}:=P \sharp Q$ as the connected sum of $P$ and $Q$.

Lemma 4.5 For each odd $n>0$ and each (sufficiently large and odd) $k>n$, the manifold $M:=B^{n} \times N^{4 k}$ has all the properties described in Lemma 4.1.

Proof The dimension of $M$ is odd and can be chosen arbitrarily large.

The manifold $N$ is simply connected, of dimension at least 5 (if $k$ is large enough) and not spin. It therefore carries a metric of positive scalar curvature (see Gromov and Lawson [12]) and the same is then true for the product $B^{n} \times N^{4 k}$.

Because $B$ is aspherical and $N$ is simply connected, we can regard the projection $p_{1}: M=B \times N \rightarrow B$ onto the first factor as the classifying map of the universal cover of $M$. By construction, the manifold $M$ has finite $\pi_{2}$ and $\pi_{4}$ and the higher $\widehat{A}$-genus $\left\langle\mathcal{A}(M) \cup \phi^{*}(c),[M]\right\rangle$ associated to the fundamental class $c \in H^{n}(B ; \mathbb{Q})=$ $H^{n}\left(B \pi_{1}(M) ; \mathbb{Q}\right)$ is nonzero. Because the group $\pi_{1}(B)$ is the fundamental group of a hyperbolic manifold, it is torsion free and does not contain $\mathbb{Z}^{2}$ as a subgroup (the latter by Preissman's theorem). This implies that the image of any homomorphism 
$\mathbb{Z} \rightarrow \operatorname{center}\left(\pi_{1}(M)\right)$ is trivial. We can therefore apply Herrera and Herrera [14, Theorem 4.1] to conclude that $M$ does not carry any effective $S^{1}$-action.

We now show that $A_{H} \subset \operatorname{Aut}\left(H_{*}(M ; \mathbb{Q})\right)$ is finite. Let $f: M \rightarrow M$ be a diffeomorphism. Up to homotopy we can assume that $f$ fixes a base point $x_{0}$ so that we get an induced automorphism $f_{*}: \pi_{1}\left(M, x_{0}\right) \rightarrow \pi_{1}\left(M, x_{0}\right)$ and together with the classifying map $p_{1}: M \rightarrow B$ a homotopy commutative diagram

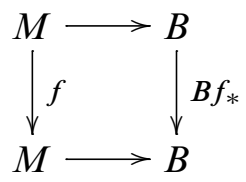

Because the automorphism $f_{*}: \pi_{1}(M) \rightarrow \pi_{1}(M)$ must be inner by our choice of $B$, the right hand vertical map induces the identity in rational cohomology. The classifying map $\phi: M \rightarrow B$ being an isomorphism in rational cohomoloy up to degree $n$ (because $k>n)$, we see that $f^{*}$ preserves the subspace $p_{1}^{*}\left(H^{*}(B ; \mathbb{Q})\right) \subset H^{*}(M ; \mathbb{Q})$ and acts as the identity on this subspace.

Up to a homotopy equivalence $\tilde{M} \rightarrow N$, the universal cover $\pi: \tilde{M} \rightarrow B \times N$ can be identified with the inclusion $N=\{*\} \times N \hookrightarrow B \times N$ (that is, the corresponding triangle diagram commutes). This holds because $B$ is aspherical and $N$ is simply connected. Hence, because the inclusion $N \hookrightarrow B \times N$ has a left inverse (take the projection $\left.p_{2}: B \times N \rightarrow N\right)$, the map $\pi$ identifies $H^{*}(\tilde{M} ; \mathbb{Q})$ with the subspace $p_{2}^{*}\left(H^{*}(N ; \mathbb{Q})\right) \subset H^{*}(B \times N ; \mathbb{Q})$. Since $f$ induces a map $\tilde{M} \rightarrow \tilde{M}$ (albeit not a map $N \rightarrow N), f^{*}$ preserves this subspace. The induced map on $H^{*}(\tilde{M} ; \mathbb{Z})$ defines an automorphism of the lattice $H^{2 k}(\tilde{M} ; \mathbb{Z})=H^{2 k}(Q ; \mathbb{Z})$. Because this lattice is positive definite, the map $f$ can induce only finitely many self maps of $H^{2 k}(\tilde{M} ; \mathbb{Q})$. The remaining nonzero rational cohomology of $\tilde{M}$ is concentrated in degrees $0, k$, $3 k$ and $4 k$ where it is isomorphic to $\mathbb{Q}$. Hence $f^{*}$ can act only by minus or plus the identity on these cohomology groups.

We conclude that $f^{*}$ preserves the subspaces $p_{1}^{*}\left(H^{*}\left(B^{n} ; \mathbb{Q}\right)\right)$ and $p_{2}^{*}\left(H^{*}(N ; \mathbb{Q})\right)$ of the vector space $H^{*}(M ; \mathbb{Q})$ and can only act as the identity on the first and in finitely many ways on the second. Because $H^{*}(M ; \mathbb{Q})$ is generated as a ring by these subspaces, $f^{*}$ is determined by the action on these subspaces. This shows that $A_{H}$ is indeed finite.

The preceding argument also shows that the induced action of $f$ on $H^{4 k}(\tilde{M} ; \mathbb{Q})$ must be the identity, since a generator of this group can be chosen as the $k$ th Pontrijagin class of $\tilde{M}$ by the construction of $Q$. This and the fact that $f^{*}$ acts trivially on $H^{n}(M ; \mathbb{Q})=$ $H^{n}\left(B^{n} ; Q\right)$ (see above) imply that $f$ must act in an orientation preserving fashion on the manifold $M$. 


\section{References}

[1] K Akutagawa, B Botvinnik, The relative Yamabe invariant, Comm. Anal. Geom. 10 (2002) 935-969 MR1957657

[2] M Belolipetsky, A Lubotzky, Finite groups and hyperbolic manifolds, Invent. Math. 162 (2005) 459-472 MR2198218

[3] A L Besse, Einstein manifolds, Ergebnisse der Mathematik und ihrer Grenzgebiete (3) 10, Springer, Berlin (1987) MR867684

[4] M Bökstedt, The rational homotopy type of $\Omega \mathrm{Wh}^{\text {Diff }}(*)$, from: “Algebraic topology, Aarhus 1982 (Aarhus, 1982)", Lecture Notes in Math. 1051, Springer, Berlin (1984) 25-37 MR764574

[5] B Botvinnik, P B Gilkey, The eta invariant and metrics of positive scalar curvature, Math. Ann. 302 (1995) 507-517 MR1339924

[6] J-P Bourguignon, Une stratification de l'espace des structures riemanniennes, Compositio Math. 30 (1975) 1-41 MR0418147

[7] GE Bredon, Sheaf theory, second edition, Graduate Texts in Mathematics 170, Springer, New York (1997) MR1481706

[8] D G Ebin, The manifold of Riemannian metrics, from: "Global Analysis (Proc. Sympos. Pure Math., Vol. XV, Berkeley, Calif., 1968)”, Amer. Math. Soc., Providence, R.I. (1970) 11-40 MR0267604

[9] F T Farrell, W C Hsiang, On the rational homotopy groups of the diffeomorphism groups of discs, spheres and aspherical manifolds, from: "Algebraic and geometric topology (Proc. Sympos. Pure Math., Stanford Univ., Stanford, Calif., 1976), Part 1", Proc. Sympos. Pure Math., XXXII, Amer. Math. Soc., Providence, R.I. (1978) 325-337 MR520509

[10] P Gajer, Riemannian metrics of positive scalar curvature on compact manifolds with boundary, Ann. Global Anal. Geom. 5 (1987) 179-191 MR962295

[11] S Goette, Morse theory and higher torsion invariants I arXiv:math.DG/0111222

[12] M Gromov, H B Lawson, Jr, The classification of simply connected manifolds of positive scalar curvature, Ann. of Math. (2) 111 (1980) 423-434 MR577131

[13] M Gromov, H B Lawson, Jr, Positive scalar curvature and the Dirac operator on complete Riemannian manifolds, Inst. Hautes Études Sci. Publ. Math. (1983) 83-196 (1984) MR720933

[14] H Herrera, R Herrera, Higher $\hat{A}$-genera on certain non-spin $S^{1}$-manifolds, Topology Appl. 157 (2010) 1658-1663 MR2639832

[15] N Hitchin, Harmonic spinors, Advances in Math. 14 (1974) 1-55 MR0358873

[16] K Igusa, Higher Franz-Reidemeister torsion, AMS/IP Studies in Advanced Mathematics 31, American Mathematical Society, Providence, RI (2002) MR1945530 
[17] K Igusa, Higher complex torsion and the framing principle, Mem. Amer. Math. Soc. 177 (2005) xiv+94 MR2155700

[18] K Igusa, Axioms for higher torsion invariants of smooth bundles, J. Topol. 1 (2008) 159-186 MR2365656

[19] A Kriegl, P W Michor, The convenient setting of global analysis, Mathematical Surveys and Monographs 53, American Mathematical Society, Providence, RI (1997) MR1471480

[20] H B Lawson, Jr, M-L Michelsohn, Spin geometry, Princeton Mathematical Series 38, Princeton University Press, Princeton, NJ (1989) MR1031992

[21] E Leichtnam, P Piazza, On higher eta-invariants and metrics of positive scalar curvature, K-Theory 24 (2001) 341-359 MR1885126

[22] J W Milnor, M A Kervaire, Bernoulli numbers, homotopy groups, and a theorem of Rohlin, from: "Proc Internat. Congress Math. 1958", Cambridge Univ. Press, New York (1960) 454-458 MR0121801

[23] S B Myers, N E Steenrod, The group of isometries of a Riemannian manifold, Ann. of Math. (2) 40 (1939) 400-416 MR1503467

[24] P Piazza, T Schick, Bordism, rho-invariants and the Baum-Connes conjecture, J. Noncommut. Geom. 1 (2007) 27-111 MR2294190

[25] J Rosenberg, $C^{*}$-algebras, positive scalar curvature, and the Novikov conjecture, Inst. Hautes Études Sci. Publ. Math. (1983) 197-212 (1984) MR720934

[26] T Schick, A counterexample to the (unstable) Gromov-Lawson-Rosenberg conjecture, Topology 37 (1998) 1165-1168 MR1632971

[27] S Stolz, Simply connected manifolds of positive scalar curvature, Ann. of Math. (2) 136 (1992) 511-540 MR1189863

[28] M Walsh, Metrics of positive scalar curvature and generalised Morse functions, part 1, Mem. Amer. Math. Soc. (2010) To appear

[29] M Walsh, Metrics of positive scalar curvature and generalised Morse functions, part 2 arXiv:0910.2114

Department of Mathematics, University of Oregon, Eugene OR 97403, USA

Institut für Mathematik, Universität Augsburg, 86135 Augsburg, Germany

Mathematisches Institut, Georg-August-Universität Göttingen, Bunsenstr. 3, 37073 Göttingen, Germany

Mathematisches Institut, WWU Münster, Einsteinstr. 62, 48149 Münster, Germany

botvinn@math.uoregon.edu, bernhard.hanke@math.uni-augsburg.de, schick@uni-math.gwdg.de, mwals_01@uni-muenster.de

Proposed: Steve Ferry

Received: 30 July 2009

Seconded: Ralph Cohen, Benson Farb

Revised: 19 October 2009 